\title{
Application of a model of plastic porous materials including void shape effects to the prediction of ductile failure under shear-dominated loadings
}

Morin, Léo; Leblond, Jean Baptiste; Tvergaard, Viggo

Published in:

Journal of the Mechanics and Physics of Solids

Link to article, DOI:

10.1016/j.jmps.2016.04.032

Publication date:

2016

Document Version

Peer reviewed version

Link back to DTU Orbit

Citation (APA):

Morin, L., Leblond, J. B., \& Tvergaard, V. (2016). Application of a model of plastic porous materials including void shape effects to the prediction of ductile failure under shear-dominated loadings. Journal of the Mechanics and Physics of Solids, 94, 148-166. https://doi.org/10.1016/j.jmps.2016.04.032

\section{General rights}

Copyright and moral rights for the publications made accessible in the public portal are retained by the authors and/or other copyright owners and it is a condition of accessing publications that users recognise and abide by the legal requirements associated with these rights.

- Users may download and print one copy of any publication from the public portal for the purpose of private study or research.

- You may not further distribute the material or use it for any profit-making activity or commercial gain

- You may freely distribute the URL identifying the publication in the public portal 


\title{
Application of a model of plastic porous materials including void shape effects to the prediction of ductile failure under shear-dominated loadings
}

\author{
Léo Morin ${ }^{1}$, Jean-Baptiste Leblond ${ }^{*}$, Viggo Tvergaard ${ }^{2}$ \\ ${ }^{1}$ Sorbonne Universités, UPMC Univ Paris 06, CNRS, UMR 7190, Institut Jean Le Rond \\ d'Alembert, F-75005, Paris, France \\ ${ }^{2}$ Department of Mechanical Engineering, Solid Mechanics, Technical University of Denmark, \\ DK-2800 Kgs. Lyngby, Denmark
}

\begin{abstract}
An extension of Gurson's famous model (Gurson, 1977) of porous plastic solids, incorporating void shape effects, has recently been proposed by Madou and Leblond (Madou and Leblond, 2012a,b, 2013; Madou et al., 2013). In this extension the voids are no longer modelled as spherical but ellipsoidal with three different axes, and changes of the magnitude and orientation of these axes are accounted for. The aim of this paper is to show that the new model is able to predict softening due essentially to such changes, in the absence of significant void growth. This is done in two steps. First, a numerical implementation of the model is proposed and incorporated into the SYSTUS ${ }^{\circledR}$ and ABAQUS ${ }^{\circledR}$ finite element programmes (through some freely available UMAT (Leblond, 2015) in the second case). Second, the implementation in SYSTUS ${ }^{\circledR}$ is used to simulate previous "numerical experiments" of Tvergaard and coworkers (Tvergaard, 2008, 2009; Dahl et al., 2012; Nielsen et al., 2012; Tvergaard, 2012, 2015a) involving the shear loading of elementary porous cells, where softening due to changes of the void shape and orientation was very apparent. It is found that with a simple, heuristic modelling of the phenomenon of mesoscopic strain localization, the model is indeed able to reproduce the results of these numerical experiments, in contrast to Gurson's model disregarding void shape effects.
\end{abstract}

Key words: Porous ductile materials, shear loadings, void shape effects, theoretical model, numerical simulations

\section{Introduction}

For many years the study of ductile failure of metals essentially concentrated on the case of high values of the triaxiality (ratio of the mean and von Mises equivalent stresses). Reviews of works in the field were provided by Tvergaard (1990) and more recently by

* Corresponding author. 
Benzerga and Leblond (2010). Most of these works found their origin in two seminal contributions: (i) that of Rice and Tracey (1969) who studied the growth of a single spherical void in an infinite plastic matrix using methods of limit-analysis, and evidenced the very strong, exponential dependence of the void growth rate upon the triaxiality; ${ }^{1}$ (ii) that of Gurson (1977) who extended Rice and Tracey (1969)'s limit-analysis to the case of a hollow sphere of finite radius, thus defining a model applicable to arbitrary, nonzero porosities.

In more recent years, increasing attention has been paid to the case of low triaxialities. Many experimental works (Bao and Wierzbicki, 2004; Barsoum and Faleskog, 2007; Beese et al., 2010; Dunand and Mohr, 2011a,b; Ghahremaninezhad and Ravi-Chandar, 2013; Haltom et al., 2013) have been devoted to the study of ductile failure under combined tension and shear, with the conclusion that it may occur even in conditions of dominant shear. Such loadings do not induce important void growth. Therefore the only possible conclusion of these observations is that changes of the shape and orientation of the voids, and especially flattening of voids subjected to shear, must induce some macroscopic softening ultimately leading to failure.

Important efforts have also been made to study ductile failure in conditions of low triaxiality via finite element micromechanical simulations of elementary porous cells subjected to loadings incorporating a shear component (Leblond and Mottet, 2008; Tvergaard, 2008, 2009; Barsoum and Faleskog, 2011; Scheyvaerts et al., 2011; Dahl et al., 2012; Nielsen et al., 2012; Tvergaard, 2012, 2015a). Among these works, those of Tvergaard and coworkers (Tvergaard, 2008, 2009; Dahl et al., 2012; Nielsen et al., 2012; Tvergaard, 2012, 2015a) appear especially significant insofar as they contained very detailed studies of the behavior of voids subjected to combined tensile and shear loadings, in conditions of possibly very low or even vanishing triaxiality, including the effect of very large deformations, possible autocontact between the voids' faces, initial void shape, etc. All these studies have shown that in conditions of dominant shear, the voids completely flatten and close up, but a mechanism of mesoscopic strain localization between voids may nevertheless occur and induce failure. When this occurs, the strain rate essentially concentrates within thin bands joining voids, whence the wording localization. However the thickness of these bands is neither zero (which would imply microscopic localization), nor of the order of the void spacing (which would imply macroscopic localization), but of the order of the void diameter, whence the wording mesoscopic localization. This mechanism may, but does not necessarily entail internal necking of intervoid ligaments leading to ultimate coalescence of voids, as commonly observed (starting with the seminal work of Koplik and Needleman (1988)) in micromechanical simulations in conditions of high triaxiality. However when the triaxiality gradually increases, there is a continuous transition between mesoscopic localization at the scale of the void diameter and internal necking of intervoid ligaments leading to coalescence.

At least for a zero triaxiality, Gurson (1977)'s model is unable to reproduce these results, even in the improved version proposed by Tvergaard and Needleman (1984) where a heuristic criterion of coalescence is introduced via some "critical" value of the porosity

$\overline{1}$ Rice and Tracey (1969)'s limit-analysis was revisited by Huang (1991), who evidenced its qualitative correctness and quantitative inaccuracy, and improved it. 
marking the onset of this phenomenon. Indeed for such a triaxiality, this model predicts no void growth; therefore the critical value of the porosity, if not reached initially, can never be reached. The origin of this failure of course lies in the fact that the model, having been developed for spherical voids only, disregards the effect of changes of the void shape and orientation. A phenomenological remedy to this situation has been proposed by Nahshon and Hutchinson (2008) in the form of a modified evolution equation of the porosity. This equation then includes an extra heuristic term depending on the three invariants of the stress tensor, which does not vanish when the triaxiality is zero and thus generates the desired softening. This very simple and effective proposal has received wide attention. It has notably been successfully applied to a DH36 steel by Xue et al. (2010), who calibrated the material parameters so as to reproduce the results of three different experiments covering a wide range of values of the triaxiality. It has also been shown by Tvergaard and Nielsen (2010) to permit satisfactory reproduction of many, if not all, results of Tvergaard and coworkers' micromechanical simulations quoted above.

The very simplicity of Nahshon and Hutchinson (2008)'s proposal however entails some inevitable shortcomings. One of these shortcomings is apparent in some very recent numerical results of Tvergaard (2015b), who compared the evolutions of elementary porous cells subjected to simple and pure shear. (Only the former type of loading had been considered in previous micromechanical simulations). These loadings generate identical overall (shear) stress states but are kinematically different. In simple shear, the principal axes of the void rotate with respect to the principal axes of the loading, and mesoscopic strain localization leading to failure is observed. In pure shear, however, there is no such rotation, and no mesoscopic strain localization occurs. ${ }^{2}$ Nahshon and Hutchinson (2008)'s proposed evolution equation of the porosity cannot account for this difference since the added term in the porosity rate depends only on the instantaneous macroscopic stress tensor, which is the same in both cases. It is thus clear that the only way to fully account for possible mesoscopic strain localization in conditions of low triaxiality is to develop a model accounting in a detailed way for the evolution of the shape and orientation of the voids.

Thus the recent interest in ductile failure at low triaxialities acts as a strong incentive to develop extensions of Gurson (1977)'s model including void shape effects. However this development in fact predates this new motivation. An extension of Gurson (1977)'s model to prolate or oblate spheroidal voids (with two distinct axes), the GLD model, ${ }^{3}$ was proposed in successive steps by Gologanu et al. (1993, 1994, 1997); Gologanu (1997). The derivation of this model was based on a generalization of Gurson (1977)'s limit-analysis of a hollow sphere to a spheroidal volume containing a confocal spheroidal void; use was made in this limit-analysis of trial velocity fields satisfying conditions of homogeneous strain rate on all spheroids confocal with the void's surface and the external boundary. ${ }^{4}$ The GLD model has notably been used by Pardoen and Hutchinson (2000) to define a refined model of void coalescence. It was recently extended to voids of arbitrary ellipsoidal

$\overline{2}$ This difference between simple and pure shear has also been noted, on completely different premises, by Song et al. (2015).

3 GLD: Gologanu-Leblond-Devaux.

4 A variant involving a velocity field orthogonal to each such spheroid was proposed by Garajeu et al. (2000). 
shape (with three different axes) by Madou and Leblond (2012a,b, 2013); Madou et al. (2013) using trial velocity fields discovered by Leblond and Gologanu (2008), satisfying conditions of homogeneous strain rate on an arbitrary family of confocal ellipsoids. The model included evolution equations of the shape and orientation of the voids borrowed, with some adaptations, from the works of Ponte-Castaneda and Zaidman (1994) and Kailasam and Ponte-Castaneda (1998), based on nonlinear homogenization theory.

The aim of this paper is to show that Madou and Leblond's model (hereafter named the $M L$ model for shortness) represents a viable, more rigorous albeit more complex, alternative to Nahshon and Hutchinson (2008)'s proposed modification of Gurson (1977)'s model, for the reproduction of the results of the micromechanical simulations of Tvergaard and coworkers of ductile failure under conditions of low triaxiality. This will serve as a first proof of the ability of the model to deal with problems of ductile rupture involving important changes of the void shape and orientation. Of course it will be necessary, as a prerequisite, to numerically implement the model into some finite element programme.

The paper is organized as follows:

- Section 2 first recapitulates the ML model. The original form of this model defined by Madou and Leblond (2012a,b, 2013); Madou et al. (2013) is envisaged first, prior to describing some heuristic extensions aimed at incorporating a few extra features, like strain hardening.

- Section 3 then proposes a finite element implementation of the model. ${ }^{5}$ This proposal makes a heavy use of Halphen and Nguyen (1975)'s and Nguyen (1977)'s definition and study of models of generalized standard materials, which possess remarkable mathematical properties bearing appealing consequences upon their numerical implementation.

- In Section 4, the proposed numerical implementation of the ML model is finally used to show that the results of the micromechanical simulations of Tvergaard and coworkers can be satisfactorily reproduced by schematizing the elementary cells considered as homogeneous volume elements obeying this model.

\section{The ML model}

\subsection{Original form}

\subsubsection{Geometrical elements}

The ML model considers, within an elastic-plastic material obeying von Mises's criterion and Prandtl-Reuss's flow rule, ellipsoidal voids of identical shape and orientation. The semi-axes $a, b, c(a>b>c)$ of these voids are oriented along unit vectors $\mathbf{e}_{x}, \mathbf{e}_{y}, \mathbf{e}_{z}$. The

\footnotetext{
5 A simpler numerical algorithm could be proposed for the homogeneous stress and strain states considered in this paper; but the finite element implementation will be compulsory for numerical simulations of full specimens and structures presented in future papers.
} 
quadratic form

$$
\mathcal{P}(\mathbf{u})=\frac{\left(\mathbf{u}_{\mathbf{e}}\right)^{2}}{a^{2}}+\frac{\left(\mathbf{u}_{\mathbf{e}} \mathbf{e}_{y}\right)^{2}}{b^{2}}+\frac{\left(\mathbf{u}_{\mathbf{e}} \mathbf{e}_{z}\right)^{2}}{c^{2}},
$$

where $\mathbf{u}$ denotes an arbitrary vector, provides the equation of the voids' surface in the form $\mathcal{P}(\mathbf{O M})=1$ where $\mathbf{O M}$ denotes the separation vector between the centre $O$ of a void and a current point $M$ on its surface. The symmetric $3 \times 3$ matrix $\mathbf{P} \equiv\left(P_{i j}\right)_{1 \leq i, j \leq 3}$ of this quadratic form in the fixed observer's basis $\left(\mathbf{e}_{1}, \mathbf{e}_{2}, \mathbf{e}_{3}\right)$ contains, in an implicit and indissociable way, all relevant information on the magnitude and orientation of the semi-axes, since its eigenvalues are $a^{-2}, b^{-2}, c^{-2}$ and the corresponding eigenvectors $\mathbf{e}_{x}$, $\mathbf{e}_{y}, \mathbf{e}_{z}$. This matrix may therefore be conveniently used in place of $a, b, c, \mathbf{e}_{x}, \mathbf{e}_{y}, \mathbf{e}_{z}$ to characterize the shape and orientation of the voids at every instant. ${ }^{6}$

The porosity (ratio of the volume of the voids over the total volume of the matter plus the voids) is classically denoted $f$.

\subsubsection{Yield criterion}

The ML macroscopic yield criterion reads

$$
\Phi(\boldsymbol{\sigma}, \mathbf{P}, f, \bar{\sigma})=\frac{\mathcal{Q}(\boldsymbol{\sigma})}{\bar{\sigma}^{2}}+2(1+g)(f+g) \cosh \left[\frac{\mathcal{L}(\boldsymbol{\sigma})}{\bar{\sigma}}\right]-(1+g)^{2}-(f+g)^{2} \leq 0 .
$$

In this equation:

- $\mathcal{Q}(\boldsymbol{\sigma})$ is a quadratic form of the (Cauchy) stress tensor $\boldsymbol{\sigma}$ given by

$$
\mathcal{Q}(\boldsymbol{\sigma})=\boldsymbol{\sigma}: \mathbf{Q}: \boldsymbol{\sigma}
$$

where $\mathbf{Q}$ is a fourth-order tensor, whose components in the principal basis $\left(\mathbf{e}_{x}, \mathbf{e}_{y}, \mathbf{e}_{z}\right)$ of the voids are given in (Madou and Leblond, 2012b).

- $\mathcal{L}(\boldsymbol{\sigma})$ is a linear form of $\boldsymbol{\sigma}$ given by

$$
\mathcal{L}(\boldsymbol{\sigma})=\kappa \mathbf{H}: \boldsymbol{\sigma}
$$

where $\kappa$ is a scalar and $\mathbf{H}$ a second-order tensor diagonal in the voids' principal basis $\left(\mathbf{e}_{x}, \mathbf{e}_{y}, \mathbf{e}_{z}\right)$, of unit trace; again, the value of $\kappa$ and the components of $\mathbf{H}$ in this basis are provided in (Madou and Leblond, 2012b).

- $g$ is the "second porosity" defined in the following way: (i) consider the completely flat ellipsoids (elliptic cracks) confocal with the ellipsoidal voids, of semi-axes $\sqrt{a^{2}-c^{2}}$, $\sqrt{b^{2}-c^{2}}, 0$, instead of $a, b, c$; (ii) rotate these flat ellipsoids about their major axis, so as to obtain prolate spheroids of semi-axes $\sqrt{a^{2}-c^{2}}, \sqrt{b^{2}-c^{2}}, \sqrt{b^{2}-c^{2}}$; (iii) consider the fictitious porosity defined by these spheroids, considered as voids. For instance, for penny-shaped cracks of semi-axes $a, a, 0, g$ is the porosity defined by fictitious spherical voids of radius $a$, often called the crack density in the relevant literature.

\footnotetext{
6 The model does not contain any characteristic lengthscale so that what in fact matters is only the ratios of two semi-axes over the third one, not their absolute values; this means that one semi-axis may conventionally be set to unity initially.
} 
- $\bar{\sigma}$ is the yield stress of the sound matrix, assumed to be constant (ideal-plasticity) in the primitive version of the model considered in this subsection.

The major novelty in the yield function defined by equation (2) with respect to that of Gurson (1977) is the appearance of the second porosity, $g$, which contributes together with the usual one, $f$, to the softening of the material. To understand why such an extra softening parameter must necessarily appear in the yield criterion, it suffices to consider the case of penny-shaped cracks. In such a case $f$ is zero, so that this parameter obviously cannot characterize the detrimental effect of the voids; the job is done instead by the crack density $g$.

It is worth noting that the yield criterion (2) has been extensively and successfully assessed by Madou and Leblond (2013), by comparing its predictions to the results of full finiteelement limit-analyses of elementary ellipsoidal cells of various shapes, including pennyshaped and elliptic cracks.

\subsubsection{Plastic flow rule}

It has been shown by Gurson (1977) that the normality of the plastic flow rule is preserved in the homogenization process used to define the models considered. Hence the flow rule reads classically

$$
\mathbf{D}^{p}=\eta \frac{\partial \Phi}{\partial \boldsymbol{\sigma}}(\boldsymbol{\sigma}, \mathbf{P}, f, \bar{\sigma}) \quad, \quad \eta\left\{\begin{array}{lll}
=0 & \text { if } & \Phi(\boldsymbol{\sigma}, \mathbf{P}, f, \bar{\sigma})<0 \\
\geq 0 & \text { if } & \Phi(\boldsymbol{\sigma}, \mathbf{P}, f, \bar{\sigma})=0
\end{array}\right.
$$

where $\mathbf{D}^{p}$ denotes the (Eulerian) plastic strain rate and $\eta$ the plastic multiplier. In this expression the derivative $\partial \Phi / \partial \boldsymbol{\sigma}$ reads, by equation (2),

$$
\frac{\partial \Phi}{\partial \boldsymbol{\sigma}}(\boldsymbol{\sigma}, \mathbf{P}, f, \bar{\sigma})=\frac{2}{\bar{\sigma}^{2}} \mathbf{Q}: \boldsymbol{\sigma}+\frac{2}{\bar{\sigma}}(1+g)(f+g) \kappa \mathbf{H} \sinh \left(\frac{\kappa \mathbf{H}: \boldsymbol{\sigma}}{\bar{\sigma}}\right) .
$$

\subsubsection{Evolution equations of the internal parameters}

It has been shown by Madou et al. (2013) that the voids being assumed to remain ellipsoidal, ${ }^{7}$ the evolution of the matrix $\mathbf{P}$ of the quadratic form $\mathcal{P}(\mathbf{u})$ characterizing their shape and orientation is governed by the equation

$$
\dot{\mathbf{P}}=-\mathbf{P} \cdot\left(\mathbf{D}^{v}+\boldsymbol{\Omega}^{v}\right)-\left(\mathbf{D}^{v}+\boldsymbol{\Omega}^{v}\right)^{T} \cdot \mathbf{P}
$$

where $\mathbf{D}^{v}$ and $\Omega^{v}$ denote the strain and rotation rate tensors of the void, respectively. The expressions of these tensors proposed by Madou et al. (2013) are basically similar to those derived by Ponte-Castaneda and Zaidman (1994) and Kailasam and Ponte-Castaneda

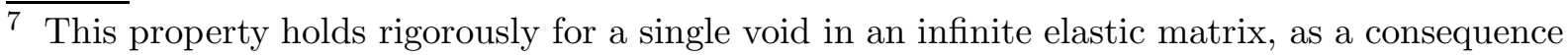
of Eshelby (1957)'s celebrated result on homogeneity of the strain in an ellipsoidal inclusion. In more complex cases it is only approximately true. 
(1998) from nonlinear homogenization theory:

$$
\left\{\begin{array}{l}
\mathbf{D}^{v}=\mathbf{L}: \mathbf{D}^{p} \\
\mathbf{\Omega}^{v}=\mathbf{\Omega}+\mathbf{R}: \mathbf{D}^{p}
\end{array}\right.
$$

where $\Omega$ is the rotation rate tensor of the material (antisymmetric part of the velocity gradient), and $\mathbf{L}$ and $\mathbf{R}$ fourth-order "localization tensors". Madou et al. (2013) however found it necessary, on the basis of numerical micromechanical simulations, to modify the expressions of these localization tensors proposed by Ponte-Castaneda and Zaidman (1994) and Kailasam and Ponte-Castaneda (1998) through introduction of heuristic corrective factors; the resulting expressions of the components of $\mathbf{L}$ and $\mathbf{R}$ in the voids' principal basis $\left(\mathbf{e}_{x}, \mathbf{e}_{y}, \mathbf{e}_{z}\right)$ are provided in (Madou et al., 2013).

The evolution equation of the porosity is classically deduced from the plastic incompressibility of the sound matrix, neglecting the small variations of this quantity arising from elasticity:

$$
\dot{f}=(1-f) \operatorname{tr} \mathbf{D}^{p}
$$

\subsection{Extensions}

The version of the model just presented is the original one proposed by Madou and Leblond (2012a,b, 2013); Madou et al. (2013). This version disregards a few phenomena which must be incorporated into the model in order to make it applicable to actual materials and situations. This is done here in a more less heuristic way, depending on the phenomenon in question.

\subsubsection{Tvergaard's parameter and mesoscopic strain localization}

In order to improve the agreement between the predictions of Gurson (1977)'s model and the results of some micromechanical simulations involving elementary cells of more realistic shape than Gurson's hollow sphere, Tvergaard (1981) proposed to modify the criterion and the associated flow rule through multiplication of the porosity $f$ by some heuristic factor $q$ slightly larger than unity. It is proposed here, for the ML model, to apply a similar multiplicative factor $q$ to the term $(f+g)$ in the yield criterion $(2)$ and the flow rule (5).

In the case of spheroidal, prolate or oblate voids, Gologanu (1997) proposed, from various arguments, to consider Tvergaard's parameter $q$ as a function of the void shape. In the general case, an arbitrary ellipsoidal void of semi-axes $a, b, c, a>b>c$ may be considered as "intermediate" between a prolate spheroidal void of semi-axes $a, c, c$ and an oblate one of semi-axes $a, a, c$; hence it is reasonable to use, for the value of $q$, an interpolation between Gologanu (1997)'s expressions for the "extreme", prolate and oblate cases. The 
expression of $q$ proposed here is of this type:

$$
q=(1-k) q^{\mathrm{prol}}+k q^{\mathrm{obl}} \quad, \quad k=\sqrt{\frac{b^{2}-c^{2}}{a^{2}-c^{2}}} \quad, \quad\left\{\begin{array}{l}
q^{\mathrm{prol}}=1+\left(q^{\mathrm{sph}}-1\right)\left[\frac{2 a / c}{1+(a / c)^{2}}\right]^{3 / 2} \\
q^{\mathrm{obl}}=1+\left(q^{\mathrm{sph}}-1\right) \frac{2 a / c}{1+(a / c)^{2}}
\end{array}\right.
$$

where $q^{\text {sph }}$ represents Tvergaard (1981)'s original value of $q$ for spherical voids, and $q^{\text {prol }}$ and $q^{\text {obl }}$ those of Gologanu (1997) for prolate and oblate spheroidal voids.

In order to account for coalescence induced by necking of intervoid ligaments, Tvergaard and Needleman (1984) proposed, in the case of spherical voids, to replace, in Gurson (1977)'s criterion and associated flow rule, the true porosity $f$ by some larger fictitious one $f^{*}$ once some "critical value" $f_{c}$ has been reached. A similar modification of the ML model is necessary to account for the phenomenon of mesoscopic strain localization discussed in the Introduction. However, in the ML model, the parameter governing softening is no longer $f$ but the sum $f+g$; hence it is natural to apply the modification to this sum and thus replace it by the quantity

$$
(f+g)^{*}=\left\{\begin{array}{llc}
f+g & \text { if } & f+g \leq(f+g)_{c} \\
(f+g)_{c}+\delta\left[f+g-(f+g)_{c}\right] & \text { if } & f+g>(f+g)_{c}
\end{array}\right.
$$

where $(f+g)_{c}$ and $\delta(>1)$ are material parameters.

With these two modifications, the expressions (2) and (6) of the yield function and its derivative become

$$
\begin{gathered}
\Phi(\boldsymbol{\sigma}, \mathbf{P}, f, \bar{\sigma})=\frac{\mathcal{Q}(\boldsymbol{\sigma})}{\bar{\sigma}^{2}}+2 q(1+g)(f+g)^{*} \cosh \left[\frac{\mathcal{L}(\boldsymbol{\sigma})}{\bar{\sigma}}\right]-(1+g)^{2}-q^{2}(f+g)^{* 2} ; \\
\frac{\partial \Phi}{\partial \boldsymbol{\sigma}}(\boldsymbol{\sigma}, \mathbf{P}, f, \bar{\sigma})=\frac{2}{\bar{\sigma}^{2}} \mathbf{Q}: \boldsymbol{\sigma}+\frac{2}{\bar{\sigma}} q(1+g)(f+g)^{*} \kappa \mathbf{H} \sinh \left(\frac{\kappa \mathbf{H}: \boldsymbol{\sigma}}{\bar{\sigma}}\right) .
\end{gathered}
$$

The model thus modified becomes the exact equivalent, for ellipsoidal voids, of the socalled GTN (Gurson-Tvergaard-Needleman) model for spherical voids.

\subsubsection{Strain hardening}

Strain hardening is introduced in exactly the same way (independent of the void shape) as in Gurson (1977)'s model:

- The sound material, instead of being ideal-plastic, is assumed to exhibit isotropic hardening, the yield stress in simple tension becoming then a known function, $\sigma(\epsilon)$, of the local cumulated equivalent strain $\epsilon$.

- The porous material is then assumed to obey the extended criterion (12), the "yield stress of the sound matrix", $\bar{\sigma}$, being now considered as identical to $\sigma(\bar{\epsilon})$ where $\bar{\epsilon}$ denotes some "average cumulated strain" of this matrix. (This means approximately replacing the true, inhomogeneously strained matrix by a fictitious, homogeneously strained one with cumulated strain $\bar{\epsilon}$ ). 
- The parameter $\bar{\epsilon}$ is finally assumed to obey Gurson (1977)'s evolution equation

$$
(1-f) \bar{\sigma} \frac{d \bar{\epsilon}}{d t}=(1-f) \sigma(\bar{\epsilon}) \frac{d \bar{\epsilon}}{d t}=\boldsymbol{\sigma}: \mathbf{D}^{p} .
$$

(This formula approximately equates the true plastic dissipation in the porous material to that in the fictitious homogeneously strained material).

\subsubsection{Contact effects}

Voids often contain quasi-rigid inclusions from which they originate; if one or several principal stresses are negative, contact of the voids' surface with the embedded inclusions may occur. Another possibility, for empty voids, is autocontact between diametrically opposed parts of the voids' surface. These effects are incorporated heuristically into the model by prescribing minimum values $a_{\min }, b_{\min }, c_{\min }$ of the voids' semi-axes; these minimum values must be taken positive to simulate contact with embedded inclusions, but nil or very small to simulate autocontact of the voids' surface. Then if, at some stage of the calculation, the value of some semi-axis, say $c$, is found to be smaller than $c_{\min }$, it is replaced by $c_{\min }$ and the found value of $f$ is correspondingly enhanced by a factor $c_{\min } / c$.

Note that although this procedure does account for the effect of contact or autocontact upon the semi-axes of the voids and the porosity, it is approximate in that it disregards its effect on the overall yield criterion and flow rule. Thus it does not represents a rigorous solution to the problem of incorporation of contact effects into the ML model, similar to Siruguet and Leblond (2004a,b)'s extension of the GLD model. ${ }^{8}$

\section{Finite element implementation of the ML model}

We concentrate here on the sole local problem of "projection", at a given integration point, of the supposedly known elastic stress predictor (elastically computed stress tensor) onto the yield locus. Other more classical aspects of the numerical implementation of such an elastic-plastic model are left aside.

\subsection{The ML model and the class of generalized standard materials}

Generalized standard materials were introduced by Halphen and Nguyen (1975) in the linearized context - small displacements and strains. This class of materials consists of

\footnotetext{
$\overline{8}$ Including contact effects into the ML model in a rigorous way would require extending the limit-analysis it originates from, by incorporating extra kinematic conditions expressing contact. This was the line followed by Siruguet and Leblond (2004a,b) for the spheroidal voids and axisymmetric loadings considered in the GLD model. The procedure was already quite involved in this simple case, and extending it to general ellipsoidal voids subjected to general loadings does not seem to be envisageable.
} 
elastic-plastic solids for which the plastic strain plus the internal parameters collectively obey some "extended normality rule". It is remarkable in several respects. The main point (Nguyen, 1977) is as follows: for such materials, the local projection problem at a given integration point, between times $t$ and $t+\Delta t$, is equivalent to minimizing some strictly convex function, provided that the flow rule is discretized with an implicit (backward Euler) scheme - that is, using the unknown values of the stresses and the internal parameters at time $t+\Delta t$ rather than the known ones at time $t$. This property guarantees existence and uniqueness of the solution of this projection problem.

It must be stressed that this property, although appealing, pertains only to the projection problem which represents only a small part of the global boundary-value problem. It says nothing about existence and uniqueness of the solution of the global problem, which are never guaranteed for softening models, such as considered in the present work.

It has been shown by Enakoutsa et al. (2007) that Gurson (1977)'s model belongs to this class, (i) in the linearized context, and (ii) provided that the sole internal parameters considered are the components of the plastic strain, $\boldsymbol{\epsilon}^{p}$, and the hardening parameter of the sound matrix, $\bar{\epsilon}$; this means artificially regarding the porosity, $f$, as a fixed quantity instead of another internal parameter. The proof relied on three properties of the model:

- the fact that strain hardening effects are summarized within a single parameter $\bar{\sigma}$, and that the yield criterion may be expressed in terms of some convex yield function of the "reduced" stress tensor $\boldsymbol{\sigma} / \bar{\sigma}$;

- the normality property of the plastic flow rule;

- the evolution equation of the hardening parameter $\bar{\epsilon}$ of the sound matrix, identical to equation (14).

Since the ML model just presented obeys the same properties, ${ }^{9}$ it may be concluded to fit into the class of generalized standard materials with the same restrictions as Gurson (1977)'s model, that is (i) in the linearized context, and (ii) artificially considering $\boldsymbol{\epsilon}^{p}$ and $\bar{\epsilon}$ as the sole internal parameters, that is regarding $f$ and the matrix $\mathbf{P}$ of the quadratic form $\mathcal{P}(\mathbf{u})$ characterizing the voids' shape and orientation as constant quantities.

In order to take advantage of the nice properties of generalized standard materials and especially of the existence and uniqueness of the solution of the projection problem, it is necessary to get rid of these two restrictions.

- With regard to large displacements and strains, their effect upon the numerical implementation in a Eulerian setting (as adopted below) reduces to two features: the equilibrium equations must be solved on the current configuration at time $t+\Delta t$, and the (hypo)elasticity law must include corrections in order to respect objectivity.

- The first feature means that at the beginning of each global elastoplastic iteration, one must update the geometry using the displacements at time $t+\Delta t$, and calculate the derivatives of the shape functions on the updated geometry. Once this is done, the geometry is fixed during the whole procedure of solution of the projection problem at

$\overline{9}$ The convexity of the ML yield function with respect to the reduced stress tensor $\boldsymbol{\sigma} / \bar{\sigma}$ has been noted by Madou and Leblond (2012b). 
each integration point. This procedure is then the same as if there was no geometry update, implying that the update has no effect upon the existence and uniqueness of the solution.

- The second feature means that the hypoelasticity law must involve, instead of the standard time-derivative of $\boldsymbol{\sigma}$, its Jaumann derivative, differing from it through combinations of $\boldsymbol{\sigma}$ and the velocity gradient. Using an explicit scheme to discretize these quantities, they become combinations of the value of $\boldsymbol{\sigma}$ at time $t$ and the gradient of the increment of displacement, $\Delta \mathbf{u}=\mathbf{u}(t+\Delta t)-\mathbf{u}(t)$. These combinations, being invariable during a given elastoplastic iteration, may be accounted for in the form of pre-corrections to the elastic predictor, added to it at the beginning of this iteration. Again, the algorithm of solution of the projection problem at each integration point is then the same as if these corrections did not exist, implying that they have no effect upon the existence and uniqueness of the solution.

- With regard to the variations of $f$ and $\mathbf{P}$, assume that the projection problem is defined with an explicit scheme with respect to these parameters, that is using their values at time $t$. They are then fixed during the entire procedure of solution of the projection problem at each integration point. Again, the projection algorithm is then the same as if $f$ and $\mathbf{P}$ were invariable in time; existence and uniqueness of the solution are guaranteed, by the properties of generalized standard materials, provided that an implicit scheme is used with respect to the parameters $\epsilon^{p}$ and $\bar{\epsilon}$.

The conclusion is as follows: in order to benefit from the existence and uniqueness of the solution of the local projection problem (again, not of the global problem) guaranteed by Halphen and Nguyen (1975)'s and Nguyen (1977)'s theory of generalized standard materials, one must respect three conditions when defining its algorithm of solution:

- in the hypoelasticity law, discretize the additional terms arising from Jaumann's stress derivative by using the value of $\boldsymbol{\sigma}$ at time $t$;

- in the projection problem, use an explicit scheme with respect to the parameters $f$ and $\mathbf{P}$

- in the same problem, use an implicit scheme with respect to the parameters $\boldsymbol{\epsilon}^{p}$ and $\bar{\epsilon}$.

\subsection{Equations of the projection problem}

The impact of large displacements and strains upon the projection problem and its algorithm of solution having just been discussed, it suffices to present them in the linearized context. The Eulerian strain rate $\mathbf{D}$ is then replaced by the time-derivative of the linearized strain $\epsilon$.

Prior to time-discretization, the equations of the "local step" (at a given integration point) 
of a given elastoplastic iteration read

$$
\begin{cases}\boldsymbol{\epsilon}=\boldsymbol{\epsilon}^{e}+\boldsymbol{\epsilon}^{p} & \text { (decomposition of the strain) } \\
\boldsymbol{\sigma}=\mathbf{C}^{e}: \boldsymbol{\epsilon}^{e} & \text { (elasticity law) } \\
\Phi(\boldsymbol{\sigma}, \mathbf{P}, f, \bar{\sigma}) \leq 0 & \text { (yield criterion) } \\
\dot{\boldsymbol{\epsilon}}^{p}=\eta \frac{\partial \Phi}{\partial \boldsymbol{\sigma}}(\boldsymbol{\sigma}, \mathbf{P}, f, \bar{\sigma}), \eta \geq 0 \quad, \quad \eta \Phi(\boldsymbol{\sigma}, \mathbf{P}, f, \bar{\sigma})=0 & \text { (plastic flow rule) } \\
\bar{\sigma}=\sigma(\bar{\epsilon}), \quad(1-f) \bar{\sigma} \frac{d \bar{\epsilon}}{d t}=\boldsymbol{\sigma}: \dot{\boldsymbol{\epsilon}}^{p} & \text { (hardening rule) } \\
\dot{\mathbf{P}}=-\mathbf{P} \cdot\left(\mathbf{D}^{v}+\boldsymbol{\Omega}^{v}\right)-\left(\mathbf{D}^{v}+\boldsymbol{\Omega}^{v}\right)^{T} \cdot \mathbf{P} & \left\{\begin{array}{l}
\text { (evolution of the voids' } \\
\text { shape and orientation) }
\end{array}\right. \\
\dot{f}=(1-f) \operatorname{tr} \dot{\boldsymbol{\epsilon}}^{p} & \text { (evolution of the porosity) }\end{cases}
$$

where $\boldsymbol{\epsilon}^{e}$ and $\boldsymbol{\epsilon}^{p}$ denote the elastic and plastic strains and $\mathbf{C}^{e}$ the elastic stiffness tensor.

Following the conclusions of the preceding discussion, equations $(15)_{1-5}$ are discretized between times $t$ and $t+\Delta t$ using an explicit scheme with respect to $f$ and $\mathbf{P}$ and an implicit one with respect to $\boldsymbol{\epsilon}^{p}$ and $\bar{\epsilon}$. Noting quantities at time $t$ with an upper index ${ }^{0}$, quantities at time $t+\Delta t$ without any index, variations of quantities between times $t$ and $t+\Delta t$ with a symbol $\Delta$, and using Voigt notations (so that second-order tensors become 6 -vectors, fourth-order tensors $6 \times 6$ matrices, and double dots simple dots), one gets the following equations defining the projection problem:

$$
\left\{\begin{array}{l}
\boldsymbol{\sigma}=\boldsymbol{\sigma}^{0}+\mathbf{C}^{e} \cdot \Delta \boldsymbol{\epsilon}^{e}=\boldsymbol{\sigma}^{\text {elast }}-\mathbf{C}^{e} . \Delta \boldsymbol{\epsilon}^{p} \quad, \quad \boldsymbol{\sigma}^{\text {elast }}=\boldsymbol{\sigma}^{0}+\mathbf{C}^{e} . \Delta \boldsymbol{\epsilon} \\
\Phi\left(\boldsymbol{\sigma}, \mathbf{P}^{0}, f^{0}, \bar{\sigma}\right) \leq 0 \\
\Delta \boldsymbol{\epsilon}^{p}=\Delta \eta \frac{\partial \Phi}{\partial \boldsymbol{\sigma}}\left(\boldsymbol{\sigma}, \mathbf{P}^{0}, f^{0}, \bar{\sigma}\right) \quad, \quad \Delta \eta \geq 0 \quad, \quad \Delta \eta \Phi\left(\boldsymbol{\sigma}, \mathbf{P}^{0}, f^{0}, \bar{\sigma}\right)=0 \\
\bar{\sigma}=\sigma\left(\bar{\epsilon}^{0}+\Delta \bar{\epsilon}\right) \quad, \quad\left(1-f^{0}\right) \bar{\sigma} \Delta \bar{\epsilon}=\boldsymbol{\sigma} \cdot \Delta \boldsymbol{\epsilon}^{p}
\end{array}\right.
$$

where $\boldsymbol{\sigma}^{\text {elast }}$ denotes the (known) elastic stress predictor, obtained by fictitiously considering the (known) increment of strain $\Delta \boldsymbol{\epsilon}$ as purely elastic.

Note that these equations do not contain the "consistency condition" $\dot{\Phi}=0$, or in discretized form $\Delta \Phi=0$, because the yield criterion is written only at time $t+\Delta t$. Thus the derivatives of the yield function with respect to the internal parameters are not required.

\subsection{Solution of the projection problem}

The treatment of strain hardening, that is the determination of $\Delta \bar{\epsilon}$ and $\bar{\sigma}$, may be achieved by a fixed point method: starting from the estimates $\Delta \bar{\epsilon}=0, \bar{\sigma}=\sigma\left(\bar{\epsilon}^{0}\right)$, one calculates $\Delta \boldsymbol{\epsilon}^{p}$ and $\boldsymbol{\sigma}$ from equations $(16)_{1-3}$, then uses equation $(16)_{4}$ to get new estimates of $\Delta \bar{\epsilon}$ and $\bar{\sigma}$, and iterates the process until convergence. This admittedly rustic method is in practice sufficient, convergence being achieved very quickly because the relative variation 
of the yield stress between successive instants of calculation is small. Thus it suffices to discuss the solution of equations $(16)_{1-3}$ for fixed values of $\Delta \bar{\epsilon}$ and $\bar{\sigma}$.

If $\Phi\left(\boldsymbol{\sigma}^{\text {elast }}, \mathbf{P}^{0}, f^{0}, \bar{\sigma}\right)<0$, the evolution between times $t$ and $t+\Delta t$ is purely elastic so that the solution is trivial: $\Delta \boldsymbol{\epsilon}^{p}=\mathbf{0}, \boldsymbol{\sigma}=\boldsymbol{\sigma}^{\text {elast }}$. We therefore henceforward concentrate on the sole non-trivial case where $\Phi\left(\boldsymbol{\sigma}^{\text {elast }}, \mathbf{P}^{0}, f^{0}, \bar{\sigma}\right) \geq 0$, implying that the criterion $\Phi\left(\boldsymbol{\sigma}, \mathbf{P}^{0}, f^{0}, \bar{\sigma}\right)=0$ must be met.

Combination of equations $(16)_{1},(16)_{3}$ and (13) then yields

$$
\boldsymbol{\sigma}=\boldsymbol{\sigma}^{\text {elast }}-\Delta \eta \mathbf{C}^{e} \cdot\left[\frac{2}{\bar{\sigma}^{2}} \mathbf{Q}^{0} \cdot \boldsymbol{\sigma}+\frac{2}{\bar{\sigma}} q^{0}\left(1+g^{0}\right)\left(f^{0}+g^{0}\right)^{*} \kappa^{0} \mathbf{H}^{0} \sinh \left(\frac{\kappa^{0} \mathbf{H}^{0} \cdot \boldsymbol{\sigma}}{\bar{\sigma}}\right)\right]
$$

which implies that

$$
\boldsymbol{\sigma}=\left[\mathbf{I}+\frac{2 \Delta \eta}{\bar{\sigma}^{2}} \mathbf{C}^{e} \cdot \mathbf{Q}^{0}\right]^{-1} \cdot \boldsymbol{\sigma}^{\text {aux }}
$$

where $\mathbf{I}$ is the unit second-rank tensor and $\boldsymbol{\sigma}^{\text {aux }}$ the "auxiliary" stress tensor given by

$$
\boldsymbol{\sigma}^{\text {aux }}=\boldsymbol{\sigma}^{\text {elast }}-\frac{2 \Delta \eta}{\bar{\sigma}} q^{0}\left(1+g^{0}\right)\left(f^{0}+g^{0}\right)^{*} \kappa^{0} \sinh \left(\frac{\kappa^{0} \mathbf{H}^{0} \cdot \boldsymbol{\sigma}}{\bar{\sigma}}\right) \mathbf{C}^{e} \cdot \mathbf{H}^{0} .
$$

Equation (17) expresses the stress $\boldsymbol{\sigma}$ as a function of $\Delta \eta$ but unfortunately also itself, since by equation (18), $\boldsymbol{\sigma}^{\text {aux }}$ depends on $\boldsymbol{\sigma}$ through the quantity $\sinh (\ldots)$. This difficulty may be obviated by considering the scalar product $\mathbf{H}^{0} . \boldsymbol{\sigma}$ as an ancillary variable; this leads to replacing equation (18) by the system

$$
\left\{\begin{array}{l}
\sigma_{h}=\mathbf{H}^{0} \cdot \boldsymbol{\sigma} \\
\boldsymbol{\sigma}^{\text {aux }}=\boldsymbol{\sigma}^{\text {elast }}-\frac{2 \Delta \eta}{\bar{\sigma}} q^{0}\left(1+g^{0}\right)\left(f^{0}+g^{0}\right)^{*} \kappa^{0} \sinh \left(\frac{\kappa^{0} \sigma_{h}}{\bar{\sigma}}\right) \mathbf{C}^{e} . \mathbf{H}^{0}
\end{array}\right.
$$

We may now show that the solution of the projection problem amounts to solving a system of two nonlinear equations on the two scalar unknowns $\Delta \eta$ and $\sigma_{h}$. The first of these equations is obtained by writing the criterion $\Phi\left(\boldsymbol{\sigma}, \mathbf{P}^{0}, f^{0}, \bar{\sigma}\right)=0$ using equations (12), (3), (4) and (17):

$$
\begin{aligned}
F( & \left.\Delta \eta, \sigma_{h}\right) \\
= & \frac{1}{\bar{\sigma}^{2}}\left\{\left[\mathbf{I}+\frac{2 \Delta \eta}{\bar{\sigma}^{2}} \mathbf{C}^{e} \cdot \mathbf{Q}^{0}\right]^{-1} \cdot \boldsymbol{\sigma}^{\mathrm{aux}}\left(\Delta \eta, \sigma_{h}\right)\right\} \cdot \mathbf{Q}^{0} \cdot\left\{\left[\mathbf{I}+\frac{2 \Delta \eta}{\bar{\sigma}^{2}} \mathbf{C}^{e} \cdot \mathbf{Q}^{0}\right]^{-1} \cdot \boldsymbol{\sigma}^{\operatorname{aux}}\left(\Delta \eta, \sigma_{h}\right)\right\} \\
& +2 q^{0}\left(1+g^{0}\right)\left(f^{0}+g^{0}\right)^{*} \cosh \left(\frac{\kappa^{0} \sigma_{h}}{\bar{\sigma}}\right)-\left(1+g^{0}\right)^{2}-q^{02}\left(f^{0}+g^{0}\right)^{* 2} \\
= & 0
\end{aligned}
$$

where the function $\boldsymbol{\sigma}^{\text {aux }}\left(\Delta \eta, \sigma_{h}\right)$ is defined by equation $(19)_{2}$. The second is obtained by writing the definition $(19)_{1}$ of $\sigma_{h}$ using again equation (17):

$$
G\left(\Delta \eta, \sigma_{h}\right)=\sigma_{h}-\mathbf{H}^{0} \cdot\left[\mathbf{I}+\frac{2 \Delta \eta}{\bar{\sigma}^{2}} \mathbf{C}^{e} \cdot \mathbf{Q}^{0}\right]^{-1} \cdot \boldsymbol{\sigma}^{\operatorname{aux}}\left(\Delta \eta, \sigma_{h}\right)=0
$$


The system of equations $(20,21)$ may be solved by a Newton method on the vectorial unknown $\left(\Delta \eta, \sigma_{h}\right)$, or two imbricated Newton methods on the scalar unknowns $\Delta \eta$ and $\sigma_{h}$. Once this is done, $\boldsymbol{\sigma}$ may be obtained from equations (17) and $(19)_{2}$, and $\Delta \boldsymbol{\epsilon}^{p}$ from equation $(16)_{3}$.

The preceding equations may also be used to calculate the constitutive tangent-matrix $\partial \boldsymbol{\sigma} / \partial \boldsymbol{\epsilon}$, from which the global consistent tangent-matrix of the elastoplastic iterations may be deduced; but this line is not pursued here. Indeed numerical experience has revealed that for the type of models envisaged here, use of the exact tangent-matrix within a rigorous Newton method is not the best method of solution for the global elastic-plastic problem anyway; the BFGS method is more robust, although it requires more iterations (but no more CPU time since no inversion of the tangent-matrix is necessary at each elastoplastic iteration).

It is worth noting that in the absence of voids, the algorithm presented above is identical to that proposed by de Borst and Feenstra (1990) and Morin et al. (2014) for Hill (1948)'s quadratic (anisotropic) criterion.

\subsection{Update of the voids' shape and orientation and the porosity}

Once the projection problem has been solved, the matrix $\mathbf{P}$ of the quadratic form $\mathcal{P}(\mathbf{u})$ characterizing the voids' shape and orientation may be updated using the following discretized version of equation $(15)_{6}$ :

$$
\Delta \mathbf{P}=-\mathbf{P}^{0} \cdot\left(\Delta \boldsymbol{\epsilon}^{v}+\Delta \boldsymbol{\omega}^{v}\right)-\left(\Delta \boldsymbol{\epsilon}^{v}+\Delta \boldsymbol{\omega}^{v}\right)^{T} \cdot \mathbf{P}^{0}
$$

in this equation, $\Delta \boldsymbol{\epsilon}^{v}$ and $\Delta \boldsymbol{\omega}^{v}$ represent the increments of deformation and rotation of the voids, given by the following equivalents of equations (8):

$$
\left\{\begin{array}{l}
\Delta \boldsymbol{\epsilon}^{v}=\mathbf{L}^{0}: \Delta \boldsymbol{\epsilon}^{p} \\
\Delta \boldsymbol{\omega}^{v}=\Delta \boldsymbol{\omega}+\mathbf{R}^{0}: \Delta \boldsymbol{\epsilon}^{p}
\end{array}\right.
$$

where $\Delta \boldsymbol{\omega}$ is the increment of rotation of the material (antisymmetric part of the gradient of the increment of displacement). (Note that a tensorial notation is again used in equations $(22,23)$ since Voigt's notation can represent neither matrix products like $\mathbf{P}^{0} . \Delta \boldsymbol{\epsilon}^{v}$, etc., nor antisymmetric tensors like $\Delta \boldsymbol{\omega}^{v}$ and $\left.\Delta \boldsymbol{\omega}\right)$.

Also, the porosity $f$ may be updated using the following discretized equivalent of equation $(15)_{7}$ :

$$
\Delta f=\left(1-f^{0}\right) \operatorname{tr} \Delta \boldsymbol{\epsilon}^{p}
$$

\subsection{Practical implementation}

The ML model has been implemented, using the algorithm just defined, in both the SYSTUS ${ }^{\circledR}$ programme developed by ESI-Group, and the ABAQUS ${ }^{\circledR}$ programme devel- 
oped by Dassault, with identical results. In the second case a UMAT (user-material subroutine) has been developed and made freely available on the Web, see the reference (Leblond, 2015). The calculations described below have been performed using SYSTUS.

\section{Application to the micromechanical simulations of Tvergaard and cowork- ers}

We shall now compare the predictions of the ML model to the results of the micromechanical simulations of Tvergaard and coworkers of elementary porous cells subjected to loadings incorporating a shear component. This will be done by applying these loadings to a mesh consisting of a single element obeying the model. We shall essentially concentrate on the simulations described in (Nielsen et al., 2012; Tvergaard, 2015a), completing the results available in these references with new ones deduced from the same simulations but not presented therein.

A general remark is that all the micromechanical simulations considered were performed using periodic, or rather quasi-periodic boundary conditions on the external surface of the cell. This implicitly meant disregarding the possible occurrence of a bifurcation mode violating periodicity. (Mesoscopic localization between voids which are not first neighbours, for instance, could generate such a mode; but such an occurrence is not very likely).

\subsection{Initially spherical voids}

* Micromechanical simulations. We consider here Nielsen et al. (2012)'s 3D simulations for initially spherical voids. The cells considered by these authors are shown schematically in Figure 1 in both their initial and deformed configurations. In the former one (Figure 1(a)), they are parallelepipedic, of sides $2 A_{0}, 2 B_{0}, 2 C_{0}$, and contain a spherical void of radius $R_{0}$. The relative dimensions are $B_{0} / A_{0}=4, C_{0} / A_{0}=1, R_{0} / A_{0}=0.5$, corresponding to an initial porosity $f_{0}=0.0164$.

The boundary conditions are as follows:

- periodic on the surfaces $x_{1}= \pm A_{0}$;

- prescribed displacements $\left(u_{1}, u_{2}, u_{3}\right)=(0,0,0)$ on the surface $x_{2}=-B_{0},\left(u_{1}, u_{2}, u_{3}\right)=$ $\left(U_{1}, U_{2}, 0\right)$ on the surface $x_{2}=B_{0}$ - these conditions are almost equivalent to periodic ones if $B_{0}$ is large enough, which is the case here;

- plane strain conditions on the surfaces $x_{3}= \pm C_{0}$.

The value of the ratio $U_{2} / U_{1}$ is continuously adjusted so as to enforce a constant value of the ratio

$$
\kappa=\frac{\Sigma_{22}}{\Sigma_{12}}
$$

where $\Sigma_{12}$ and $\Sigma_{22}$ represent overall stress components, obtained through averaging of the 


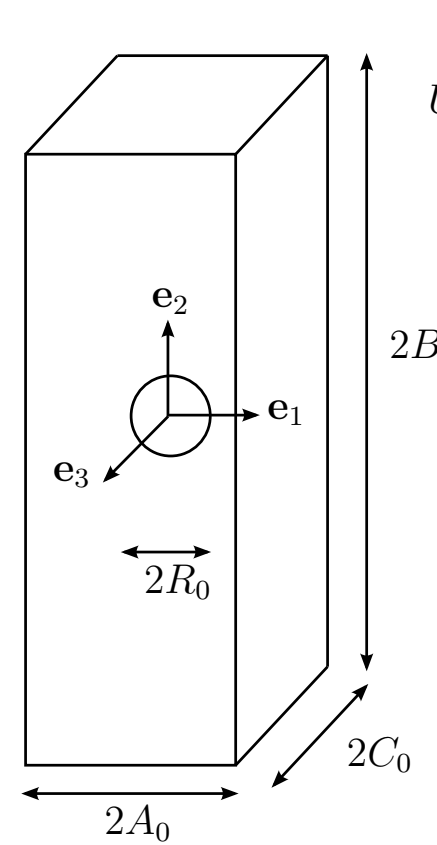

(a) Initial configuration

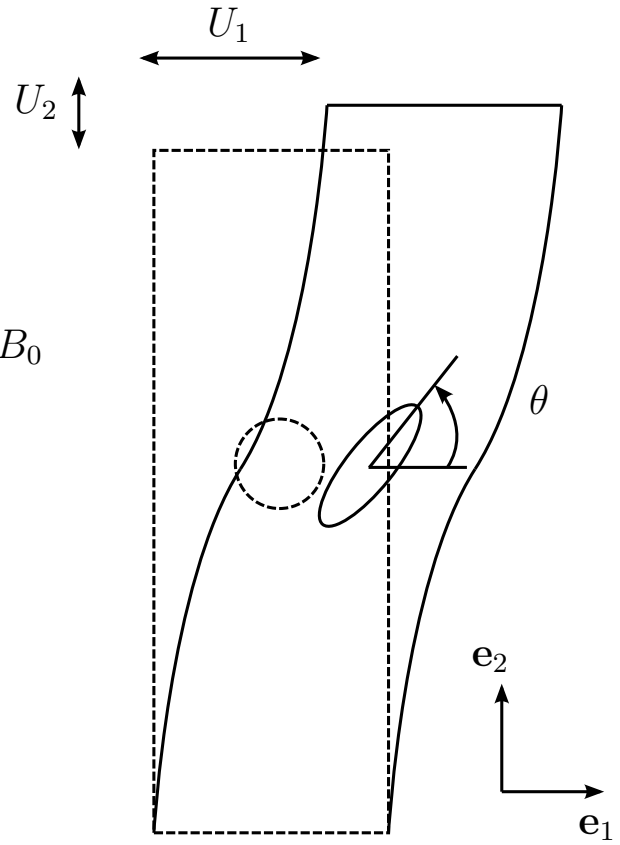

(b) Deformed configuration

Fig. 1. Elementary cell containing an initially spherical void (after Nielsen et al. (2012)) corresponding local components. ${ }^{10}$ Possible autocontact between diametrically opposed parts of the void's surface is accounted for through some penalty method.

The material parameters are as follows: Young's modulus, $E=200 \mathrm{GPa}$; Poisson's ratio, $\nu=0.3$; initial yield stress, $\sigma_{0}=400 \mathrm{MPa}$; isotropic hardening with exponent $n=0.2$.

The quantities considered include the overall shear stress $\Sigma_{12}$, the porosity $f$, the inclination of the void characterized by the angle $\theta$ defined in Figure 1(b), and the semi-axes $R_{i}$ normalized by their initial value $R_{0}$. These quantities are plotted versus the "shear angle"

$$
\Psi=\arctan \frac{U_{1}}{2 B_{0}+U_{2}},
$$

for various values of the ratio $\kappa$ defined above.

* Simulations based on the ML model. To mimic Nielsen et al. (2012)'s simulations with the ML model, we use the following parameters: Tvergaard's parameter for a spherical void, $q^{\text {sph }}=1.47$ - value recommended by Perrin and Leblond (1990) from a "self-consistent" approach; parameters for mesoscopic strain localization, see Table 1 below.

* Comparison of results. Figure 2 displays the comparison between the results of the two types of simulations. ${ }^{11}$ For the overall shear stress $\Sigma_{12}$ (Figure 2(a)), the theoretical predictions reproduce the numerical results very well, including the final decrease of the

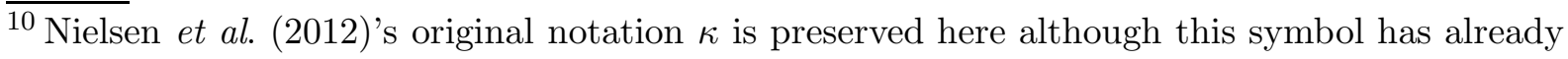
been used in the expression (4) of the linear form $\mathcal{L}(\boldsymbol{\sigma})$; there is no risk of confusion since expression (4) will play no role in the rest of the paper. Also, capital letters are used here to symbolize the averaging process, but $\Sigma_{12}$ and $\Sigma_{22}$ simply correspond to the local stress components $\sigma_{12}$ and $\sigma_{22}$ in the ML homogenized model.

${ }^{11}$ In all figures the angle $\Psi$ characterizing the overall transformation gradient is measured in
} 
Table 1

\begin{tabular}{c|cccccc}
$\kappa$ & 1.5 & 1.25 & 1 & 0.75 & 0.5 & 0.25 \\
\hline$(f+g)_{c}$ & 0.032 & 0.035 & 0.039 & 0.043 & 0.047 & 0.051 \\
$\delta$ & 8 & 8 & 8 & 8 & 8 & 8
\end{tabular}

Parameters of mesoscopic strain localization for an initially spherical void - $f_{0}=0.0164$

stress due to mesoscopic strain localization. This good agreement is admittedly achieved by allowing the parameter $(f+g)_{c}$ to slightly depend upon the load parameter $\kappa$, see Table 1. But the necessity of such a dependence upon the loading has already been noted by Koplik and Needleman (1988) and Perrin (1992) for the simpler GTN model, in the absence of macroscopic shear, and obviously arises from the crudeness of the modelling of mesoscopic strain localization.

The model also reproduces the evolution of the porosity $f$ (Figure 2(b)) rather well, including its slight decrease for small values of $\kappa$ (small axial stress $\Sigma_{22}$ ).

Finally the evolutions of the orientation of the void (Figure 2(c)) and its semi-axes (Figures $2(\mathrm{~d})$ and 2(e)) are acceptably reproduced, although the model tends to minimize the void's change of shape. The quality of the model predictions is however certainly impaired here by the fact that the "numerical void" does not remain strictly ellipsoidal when deformed, especially for low triaxialities. Indeed in such conditions it often approximately takes the shape of a "dumbbell", for which contact between diametrically opposed parts of the surface occurs earlier than for an ellipsoid - note that in Figure 2(e) for $\kappa=0.25$, the minor semi-axis $c$ becomes zero (implying contact) in the micromechanical simulation at about $\Psi=0.8$, when the value predicted by the model is still positive. It is also interesting to note that the three semi-axes take quite distinct values, which illustrates the difficulty of applying the simpler GLD model, based on the assumption of two distinct axes only, to the situations considered here.

\subsection{Initially circular cylindrical voids}

* Micromechanical simulations. Nielsen et al. (2012) have also performed 2D, plane strain simulations analogous to the preceding 3D ones, but for initially circular cylindrical voids instead of spherical ones. The initial cell geometry considered is shown in Figure 3. The value of the ratio $R_{0} / A_{0}$ of the initial radius $R_{0}$ of the void to the horizontal half-dimension $A_{0}$ of the cell is changed to 0.29 so as to warrant an (almost) identical initial porosity, $f_{0}=0.0165$.

* Simulations based on the ML model. To tentatively reproduce the numerical results with the ML model, we use the same value of Tvergaard's parameter for a spherical void as before, $q^{\text {sph }}=1.47$, but slightly different parameters for mesoscopic strain localization, see Table 2 below.

radians; but in Figure 2(c) and similar ones the angle $\theta$ characterizing the orientation of the void is measured in degrees. 


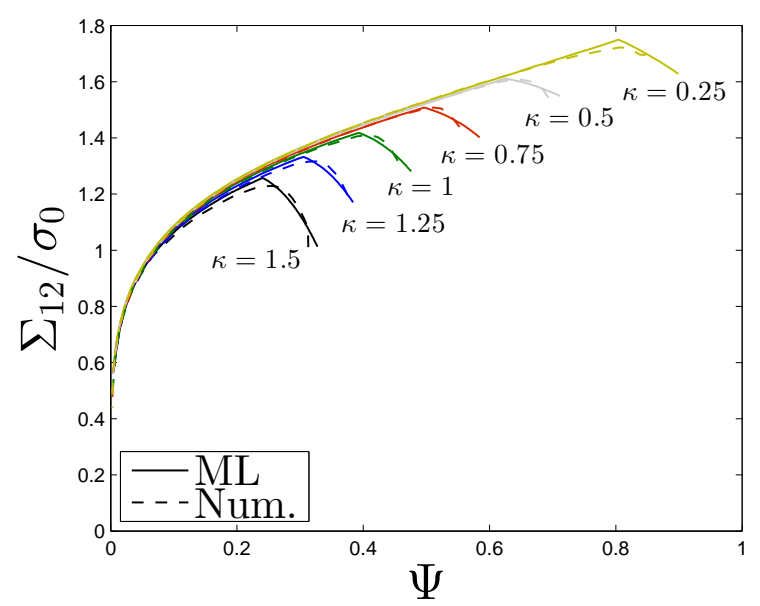

(a) Normalized overall shear stress

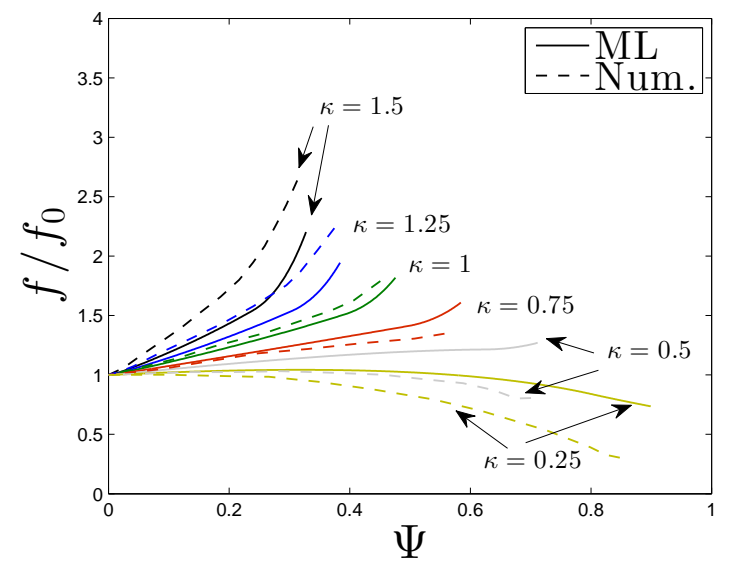

(b) Normalized porosity

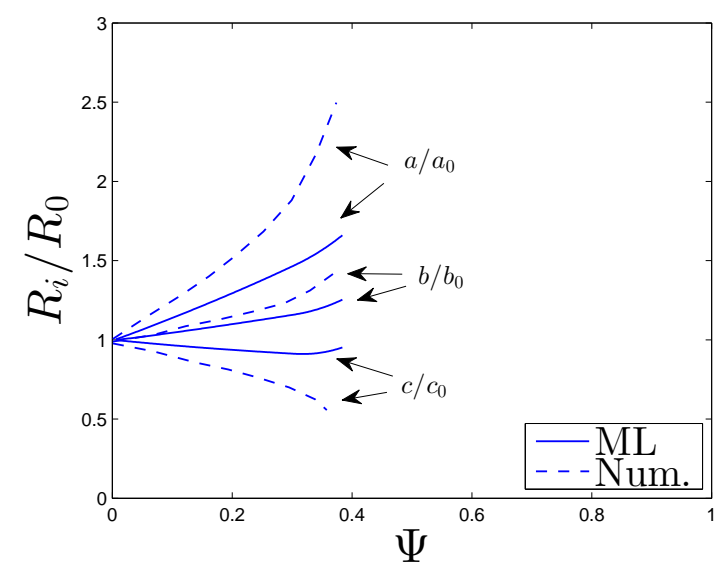

(d) Normalized semi-axes $(\kappa=1.25)$

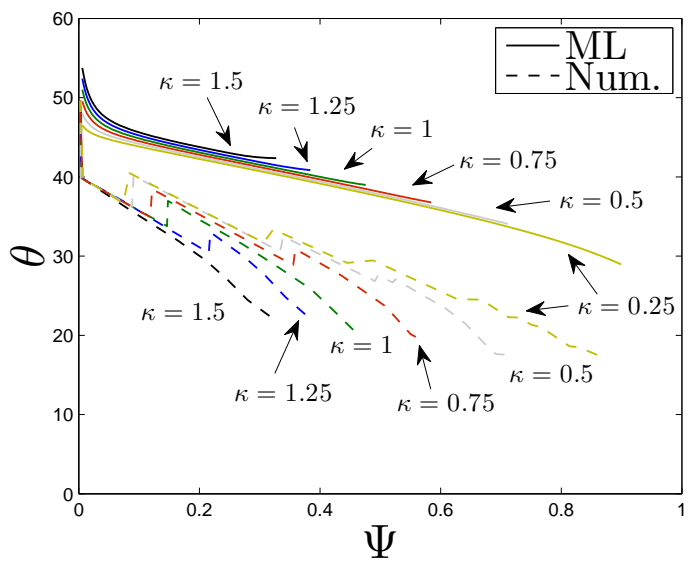

(c) Void orientation

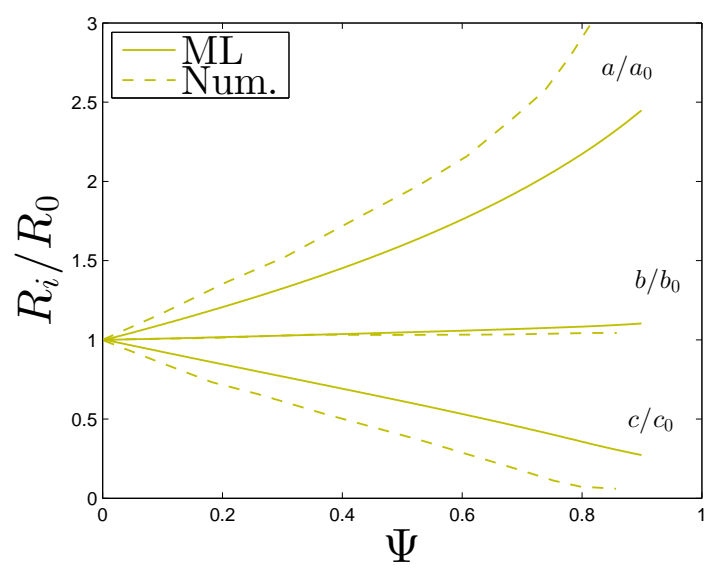

(e) Normalized semi-axes $(\kappa=0.25)$

Fig. 2. Comparison of numerical results and model predictions - Initially spherical void, $f_{0}=0.0164$

* Comparison of results. Figure 4 displays the comparison of micromechanical results and model predictions. The overall shear stress $\Sigma_{12}$ (Figure 4(a)) is again very well reproduced by the model. The porosity $f$ (Figure $4(\mathrm{~b})$ ) is somewhat less well reproduced than for an initially spherical void (compare to Figure 2(b)), but global tendencies - notably the 


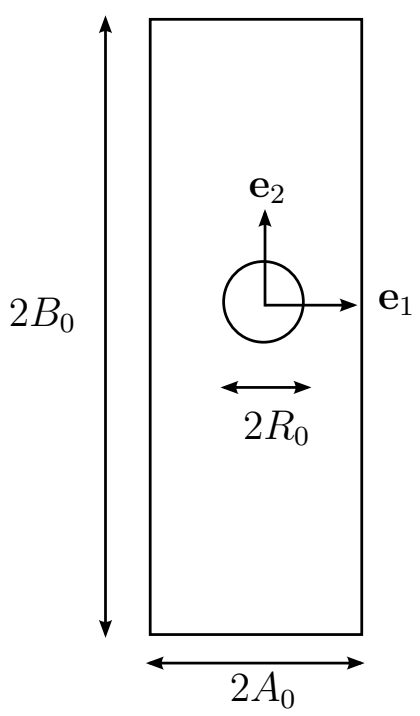

Fig. 3. Elementary cell containing an initially circular cylindrical void (after Nielsen et al. (2012))

\begin{tabular}{c|cccccc}
$\kappa$ & 1.5 & 1.25 & 1 & 0.75 & 0.5 & 0.25 \\
\hline$(f+g)_{c}$ & 0.035 & 0.04 & 0.047 & 0.058 & 0.068 & 0.08 \\
$\delta$ & 15 & 15 & 15 & 15 & 15 & 15
\end{tabular}

Table 2

Parameters of mesoscopic strain localization for an initially circular cylindrical void - $f_{0}=0.0165$

dependence on $\kappa$ - are nevertheless correctly captured.

For the orientation of the void (Figure 4(c)) and the values of its semi-axes (Figures 4(d) and $4(\mathrm{e})$ ), the model predictions are of the same overall quality as for an initially spherical void - compare to Figures 2(c), 2(d) and 2(e). Again, the variations of the semi-axes are somewhat underestimated by the model, and in Figure 4(e) for $\kappa=0.25$, the value of the minor semi-axis $c$ predicted by the model is still positive at about $\Psi=0.4$, when contact occurs in the micromechanical simulation. The common explanation of these discrepancies probably again lies in the fact that the cross-section of the numerical void becomes in time more "dumbbell-shaped" than elliptic.

\subsection{Initially elliptic cylindrical voids}

* Micromechanical simulations. We finally consider 2D, plane strain simulations of Tvergaard (2015a) for initially elliptic cylindrical voids. These simulations are especially interesting in that they evidence a strong effect of the initial void shape. The initial cell geometry is the same as before, but now $B_{0} / A_{0}=2$ instead of 4 , and the void is an elliptic cylinder of semi-axes $R_{10}$ and $R_{20}$ (Figure 5(a)). Various values of the void aspect ratio

$$
\alpha=\frac{R_{10}}{R_{20}}
$$

are envisaged. The initial porosity is $f_{0}=0.02452$ in all cases. 


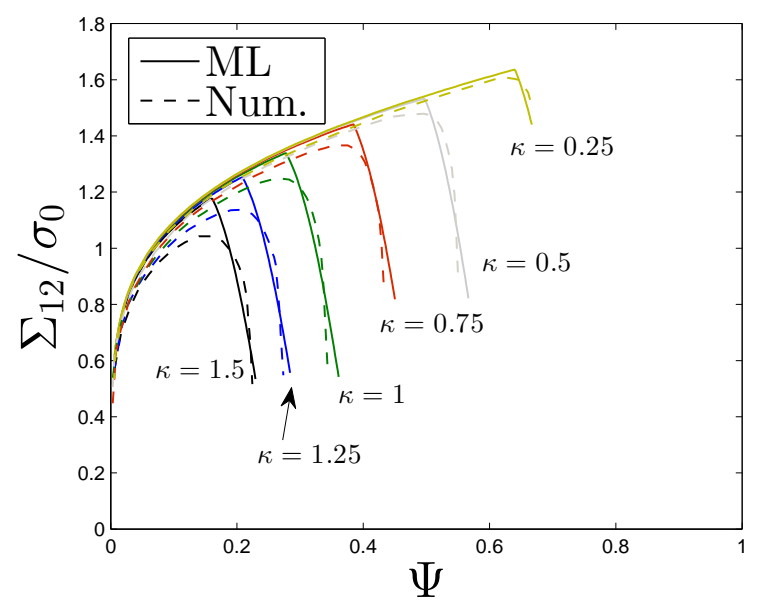

(a) Normalized overall shear stress

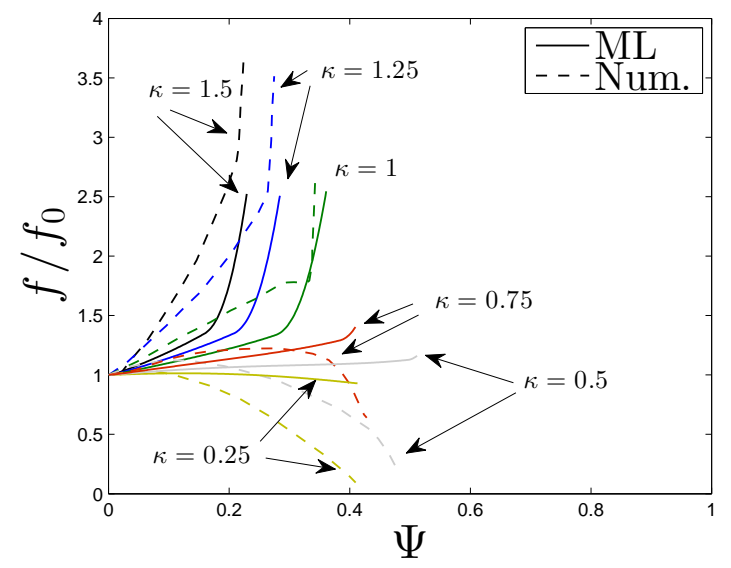

(b) Normalized porosity

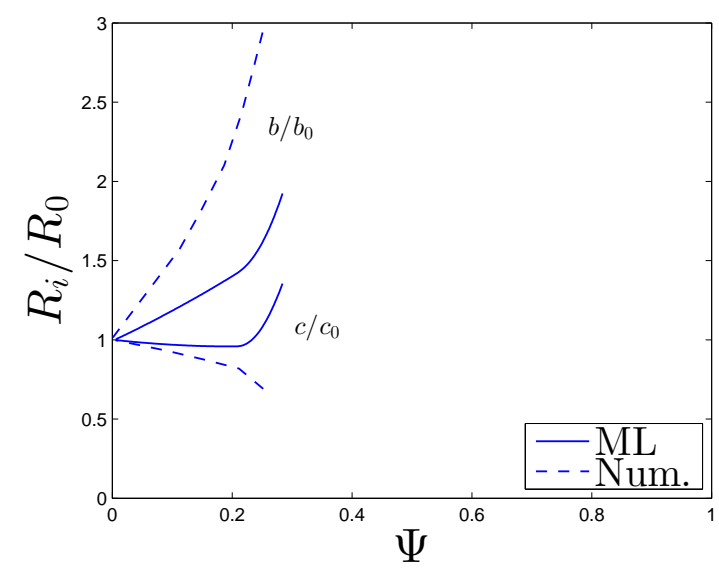

(d) Normalized semi-axes $(\kappa=1.25)$

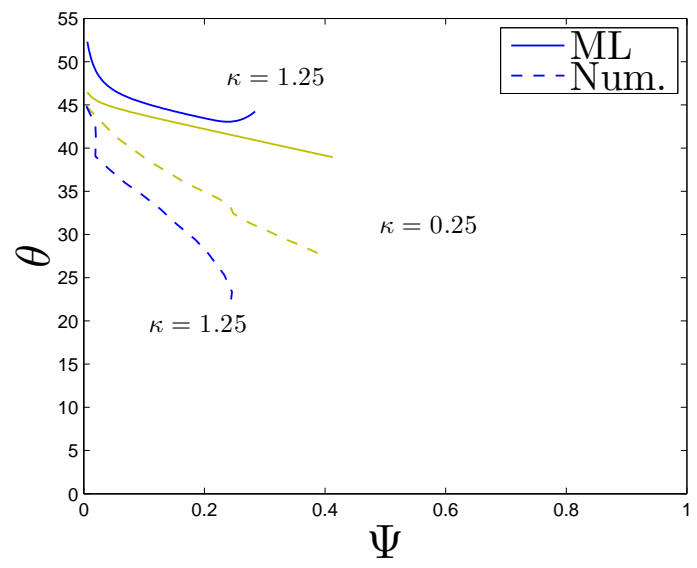

(c) Void orientation

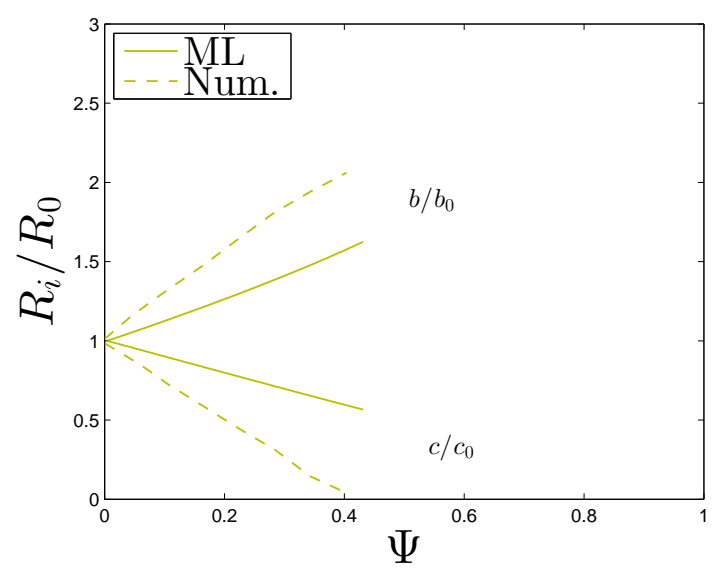

(e) Normalized semi-axes $(\kappa=0.25)$

Fig. 4. Comparison of numerical results and model predictions - Initially circular cylindrical void, $f_{0}=0.0165$

The boundary conditions are now fully periodic on all bounding surfaces of the cell, $x_{1}= \pm A_{0}, x_{2}= \pm B_{0}$. The overall displacements imposed $U_{1}^{\text {top }}, U_{1}^{\text {bot }}, U_{2}$ (see Figure $5(\mathrm{~b}))$ are continuously adjusted so as to enforce constant values of the ratios $\Sigma_{11} / \Sigma_{12}$ and $\Sigma_{22} / \Sigma_{11}$; in practice $\Sigma_{11} / \Sigma_{12}=0.1$ and $\Sigma_{22} / \Sigma_{11}=3$ (so that $\kappa=\Sigma_{22} / \Sigma_{12}=0.3$ ). 


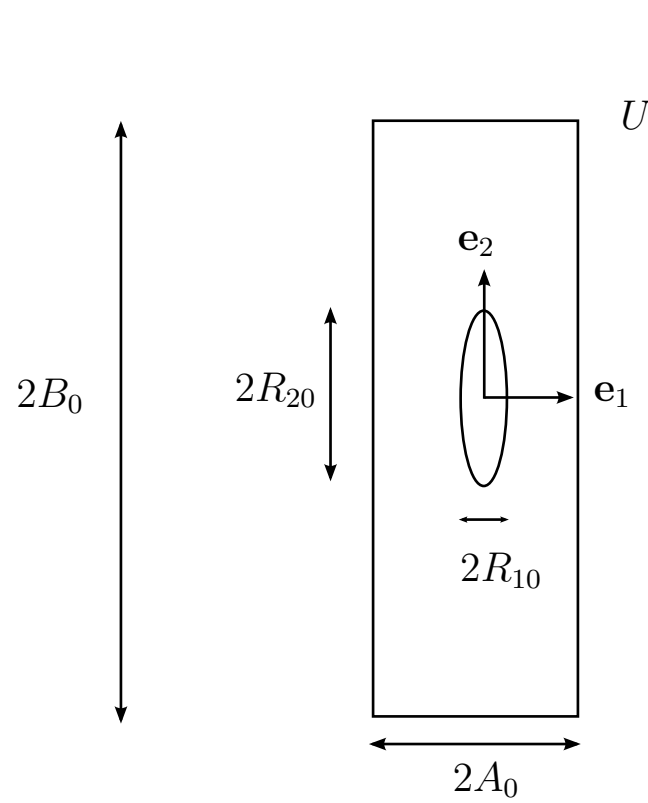

(a) Initial configuration

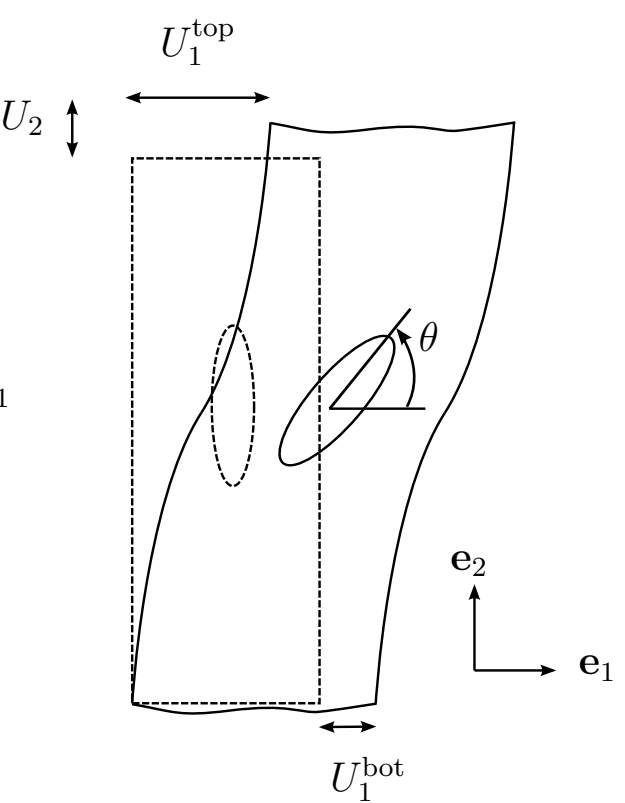

(b) Deformed configuration

Fig. 5. Elementary cell containing an initially elliptic cylindrical void (after Tvergaard (2015a))

Possible autocontact between diametrically opposed parts of the void's surface is accounted for here in a simplified way, by introducing an internal pressure into the void, perpendicularly to its major axis, when the ratio of its minor axis to its major one becomes smaller than some prescribed value (in practice 0.15). Numerical studies of Dahl et al. (2012) have shown that this approximate procedure is acceptable.

The values of the material parameters are the same as before except for the hardening exponent, for which the value $n=0.1$ is now used instead of 0.2 .

* Simulations based on the ML model. To reproduce the numerical results with the ML model, we again use the value $q^{\text {sph }}=1.47$, but slightly different values of $(f+g)_{c}$ and $\delta$, now depending on the void aspect ratio $\alpha$, see Table 3 below.

Table 3

\begin{tabular}{c|ccccc}
$\alpha$ & 0.25 & 0.5 & 1 & 2 & 4 \\
\hline$(f+g)_{c}$ & 0.16 & 0.13 & 0.095 & 0.074 & 0.105 \\
$\delta$ & 15 & 15 & 15 & 9 & 8
\end{tabular}

Parameters of mesoscopic strain localization for an initially elliptic cylindrical void - $f_{0}=0.02452$

In addition, since the minor semi-axis of the void is prevented from going to zero in the micromechanical simulations, a positive minimum value $c_{\min }$ of this semi-axis is introduced into the model. This value is chosen so as to be compatible with the minimum value of 0.15 of the ratio of the minor to major semi-axes imposed by Tvergaard (2015a).

* Comparison of results. Figures 6 and 7 compare numerical results to model predictions; all quantities are plotted here versus the shear component of the overall transformation gradient, $F_{12}=U_{1}^{\text {top }} /\left(2 B_{0}\right)$. Note that in Figures $6(\mathrm{~b}), 6(\mathrm{c})$ for the porosity and the void's orientation and $7(\mathrm{a})-7(\mathrm{e})$ for the void's semi-axes, the curves are often stopped earlier 
than in Figure 7(a) for the overall shear stress. The reason is that since (in both the micromechanical simulations and the model) the internal pressure is imposed before the void's minor axis becomes truly zero, its introduction certainly impairs the subsequent evolutions of the porosity and the minor semi-axis, perhaps also those of the major semiaxis and the orientation of the void; therefore the curves representing these evolutions are truncated when (pseudo-)contact sets in.

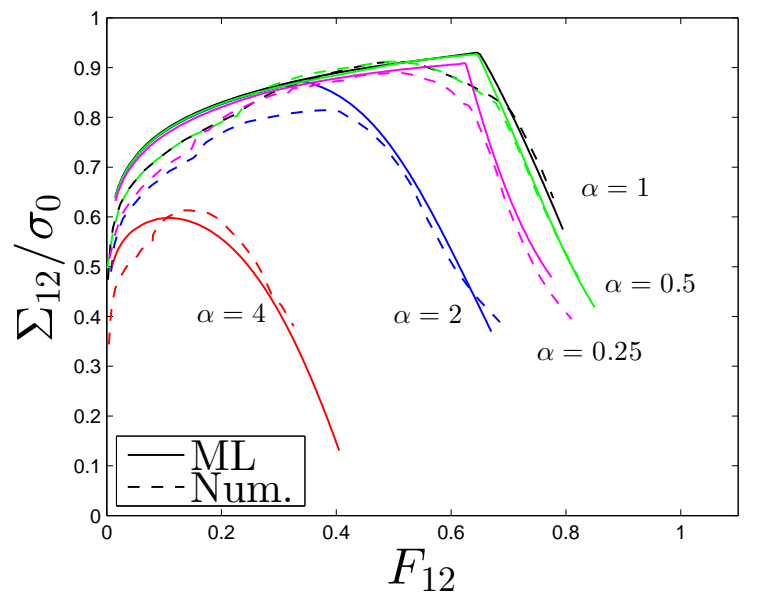

(a) Normalized overall shear stress

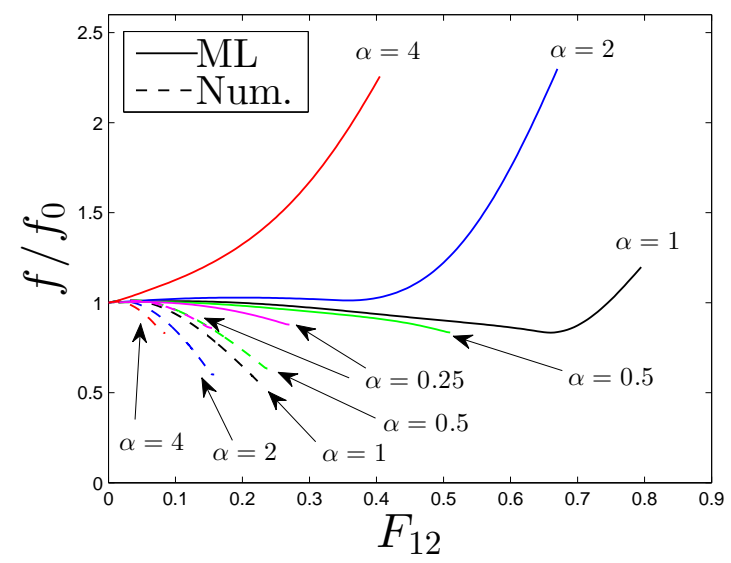

(b) Normalized porosity

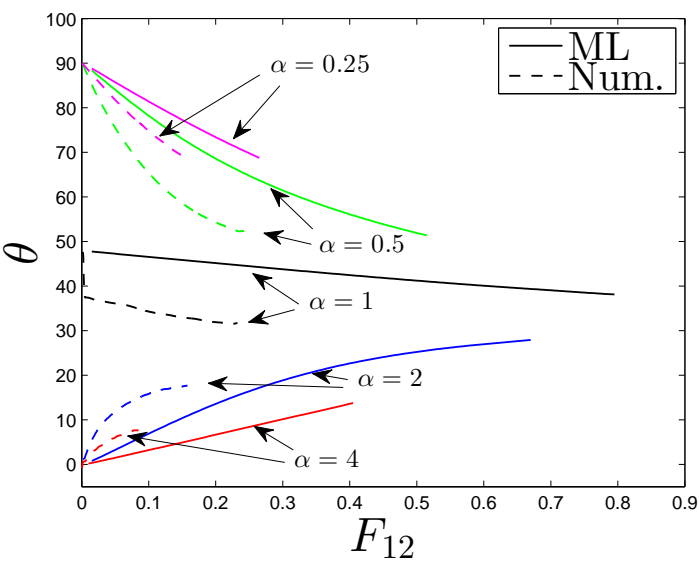

(c) Void orientation

Fig. 6. Comparison of numerical results and model predictions - Initially elliptic cylindrical void, $f_{0}=0.02452$

For the overall shear stress $\Sigma_{12}$ (Figure 6(a)), the agreement is again very good. The reproduction by the model of the large influence of the void aspect ratio $\alpha$, on both the behavior prior to mesoscopic strain localization and the onset of localization, is especially remarkable. Clearly, and appealingly, the void with initially circular cross-section is the least detrimental; and for the voids with elliptic cross-section, these with initial major axis oriented in the horizontal direction of shear, that is having $R_{10}>R_{20}$ or $\alpha>1$, are more detrimental than those with initial major axis oriented in the perpendicular vertical direction, that is having $R_{10}<R_{20}$ or $\alpha<1$.

For the porosity $f$ (Figure 6(b)), the agreement of numerical results and theoretical predictions is notably poorer than for initially spherical or circular cylindrical voids (compare 


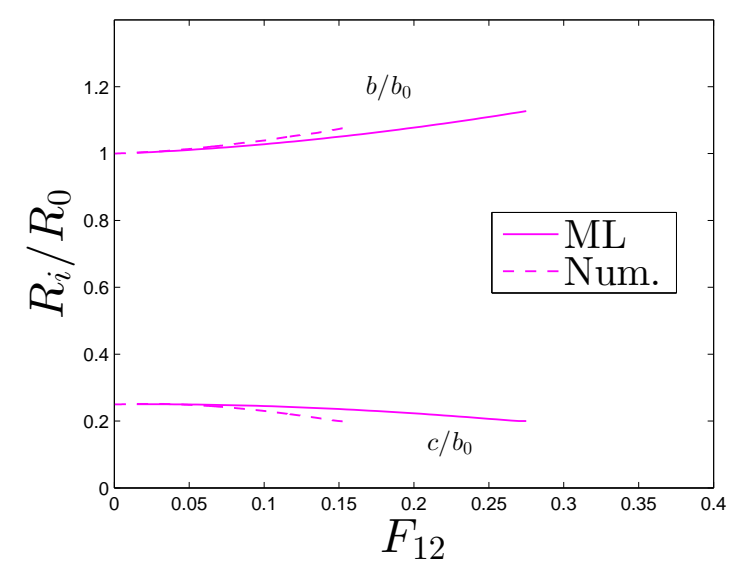

(a) Normalized semi-axes $(\alpha=0.25)$

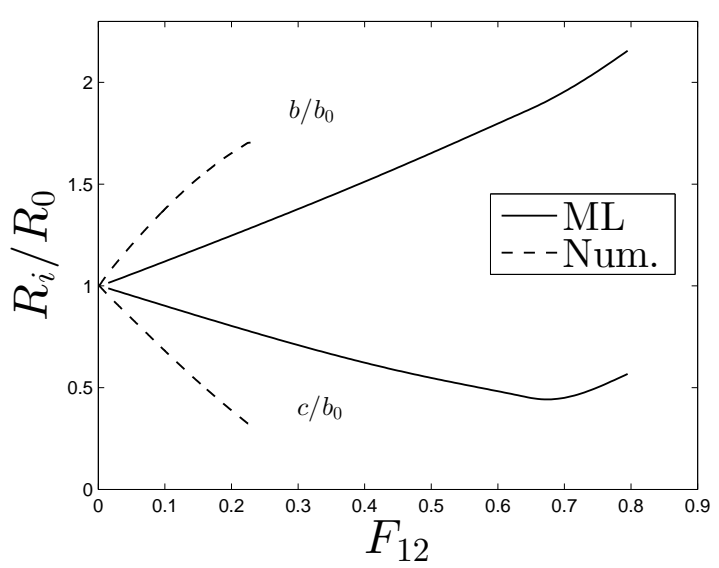

(c) Normalized semi-axes $(\alpha=1)$

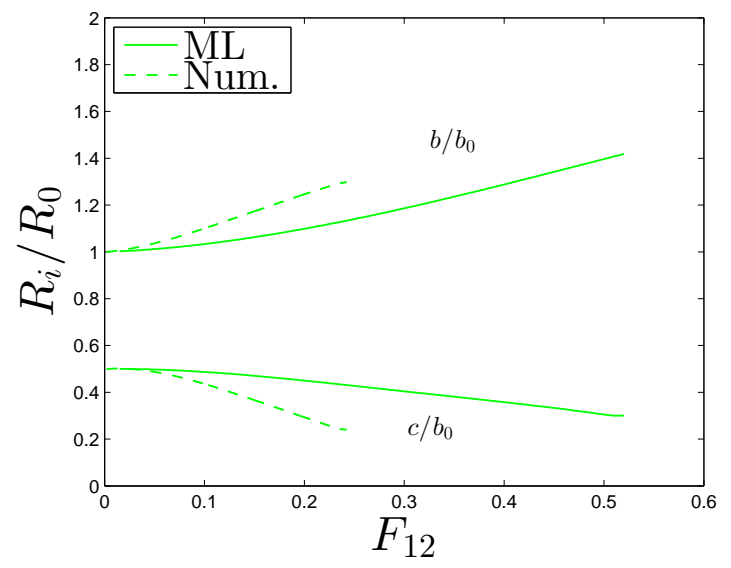

(b) Normalized semi-axes $(\alpha=0.5)$

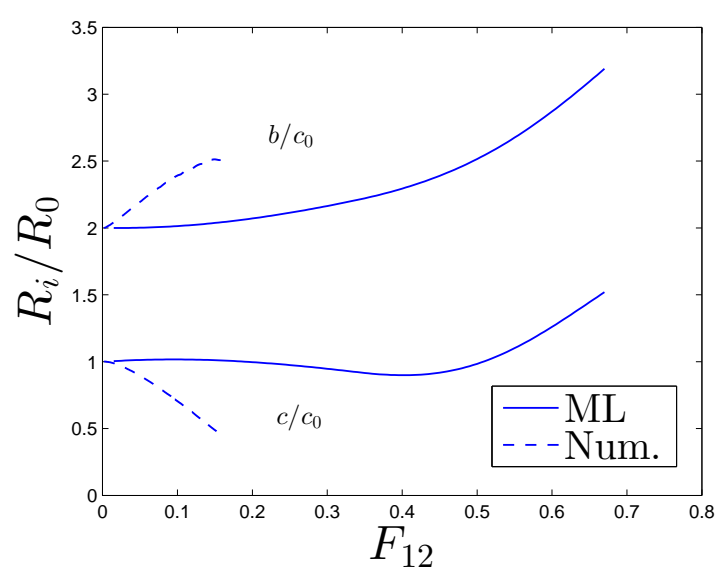

(d) Normalized semi-axes $(\alpha=2)$

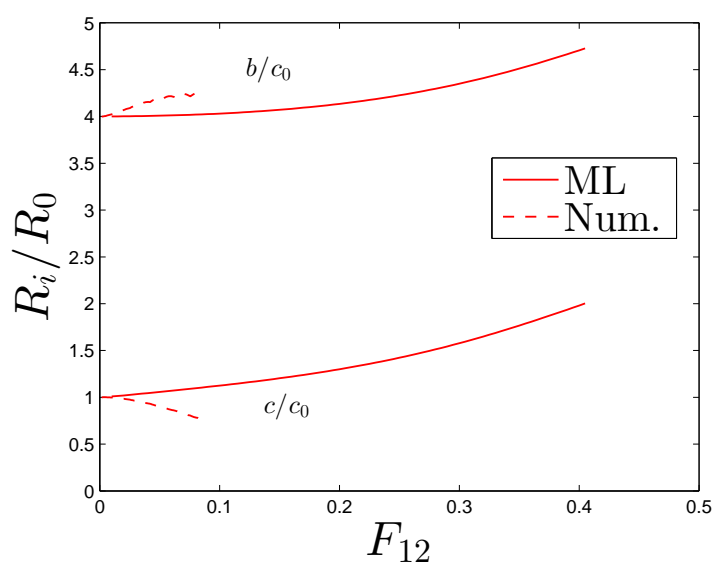

(e) Normalized semi-axes $(\alpha=4)$

Fig. 7. Comparison of numerical results and model predictions - Initially elliptic cylindrical void, $f_{0}=0.02452$

to Figures 2(b) and 4(b)). Note for instance that for $\alpha=2$ and 4, the numerical porosity slightly decreases while the theoretical one unrealistically increases. ${ }^{12}$ But these discrep-

$\overline{12}$ This is probably because the heuristic modelling of mesoscopic strain localization through replacement of $f+g$ with $(f+g)^{*}$ tends to enhance the porosity rate. 
ancies have little impact upon the quality of the model predictions for the overall shear stress.

Finally for the orientation of the void (Figure 6(c)), the model again makes an acceptable job, whereas for the values of the semi-axes (Figures 7(a) - 7(e)), the model predictions are somewhat less satisfactory. It is worth noting in this respect that in the micromechanical simulations contact occurs for all values of $\alpha$, whereas the model predicts that contact occurs only for $\alpha=0.25$ and 0.5 , and for larger values of $F_{12}$ than in the micromechanical simulations $(0.27$ versus 0.16 for $\alpha=0.25,0.5$ versus 0.25 for $\alpha=0.5)$. The fact that the model predicts less contact than actually observed numerically is a consequence of the errors due to the hypothesis of persistent ellipsoidal shape of the void; and small values of $\alpha$ favor contact in the model because the smaller this parameter, the smaller the initial distance between the quasi-vertical parts of the void's surface which will ultimately come in contact. ${ }^{13}$

\subsection{Discussion}

It is useful here to distinguish between the phases preceding and following the onset of mesoscopic localization; indeed these phases are different from a modelling point of view since the heuristic parameters $(f+g)_{c}$ and $\delta$ do not have any impact upon the first one, but play a role during the entire second one.

During the phase preceding the onset of mesoscopic localization, the model predictions do not involve any adjustable parameter. They reproduce the influence of the initial void shape in a remarkable way. They do predict that the softening effect of the void is somewhat stronger for an initially circular cylindrical void than for an initially spherical one (compare Figures 2(a) and 4(a)). More importantly, for elliptic cylindrical voids, the ML model captures very well the important influence of the void aspect ratio, the detrimental effect of the void being maximal when its cross-section is an elongated ellipse with major axis oriented in the direction of shear (Figure 6(a)).

With regard to the phase following the onset of mesoscopic localization, the ML model correctly predicts both the onset of mesoscopic localization and the subsequent evolutions of quantities. It may be objected that this success is obtained at the expense of (i) introduction of the heuristic parameters $(f+g)_{c}$ and $\delta$, and (ii) adjustment of these parameters as functions of the loading and, in the case of elliptic cylindrical voids, the void aspect ratio. One may answer to the first objection that Gurson (1977)'s model, even if taken in the form of the GTN variant incorporating analogous parameters $f_{c}$ and $\delta$, cannot do a similar job since it predicts softening only if the porosity increases, which is not the case in several of the micromechanical simulations. And the natural answer to the second objection is that the necessary variations of the parameters $(f+g)_{c}$ and $\delta$, which were already observed by Koplik and Needleman (1988) and Perrin (1992) in the classical case

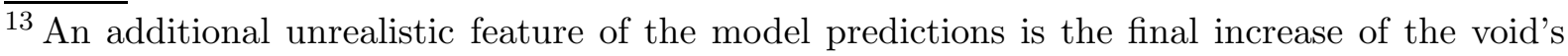
minor semi-axis $c$ for $\alpha=1$ and 2, probably again due to the crudeness of the heuristic modelling of mesoscopic strain localization.
} 
of initially spherical voids subjected to axisymmetric loadings, are a consequence of the crude modelling of mesoscopic strain localization; it is hoped that more rigorous models of this phenomenon, based on micromechanical analyses extending that recently proposed by Benzerga and Leblond (2014), will be developed in the future and incorporated into the model.

The model predicts occurrence of contact between diametrically opposed parts of the void's surface less frequently than what is actually observed in the micromechanical simulations. This is due to the hypothesis it makes of persisting ellipsoidal shape, which disregards the thinning of the central part of the void leading to easier and earlier contact. But this discrepancy does not seem to bear important consequences upon the quality of the model predictions with regard to the overall stresses and the global softening.

A comparison with Nahshon and Hutchinson (2008)'s proposed heuristic modelling of ductile damage in shear within Gurson (1977)'s model is finally in order. The simplicity and practical usefulness of Nahshon and Hutchinson (2008)'s proposal are not in question here. It is recalled in particular that Tvergaard and Nielsen (2010) have shown that it permits, with a suitable adjustment of a parameter governing the growth of the "porosity" in shear, to satisfactorily reproduce the results of their micromechanical simulations of initially spherical and circular cylindrical voids. But such a good agreement would not be obtained for Tvergaard (2015a)'s simulations of initially elliptic cylindrical voids, since they evidence a large effect of the initial void shape which is not incorporated in Gurson (1977)'s model, even with Nahshon and Hutchinson (2008)'s modified evolution equation of the porosity.

\section{$5 \quad$ Summary and perspectives}

The aim of this paper was to assess the capabilities of the ML model of porous plastic solids recently developed by Madou and Leblond (2012a,b, 2013); Madou et al. (2013), extending Gurson (1977)'s classical model through incorporation of void shape effects. This was done by comparing the predictions of this model to the results of some micromechanical simulations, used as references, of Tvergaard and coworkers (Tvergaard, 2008, 2009; Dahl et al., 2012; Nielsen et al., 2012; Tvergaard, 2012, 2015a) of elementary porous cells subjected to various loadings including an important shear component.

In a first step, a finite element implementation of the model was proposed. Heavy use was made there of the theory of generalized standard materials developed by Halphen and Nguyen (1975) and Nguyen (1977). This theory served as a guide to define an implementation ensuring existence and uniqueness of the solution of the local problem, at an arbitrary integration point, of "projection" of the (supposedly known) elastic stress predictor onto the complex ML yield locus. This implementation has been incorporated into the SYSTUS ${ }^{\circledR}$ and ABAQUS ${ }^{\circledR}$ finite element programmes; for the latter programme this has been done by writing a UMAT made freely available on the Web (Leblond, 2015).

In a second step, the micromechanical simulations of Nielsen et al. (2012); Tvergaard (2015a) were tentatively reproduced by applying the loadings considered by these authors 
to a mesh consisting of a single element obeying the ML model. All results of Nielsen et al. (2012); Tvergaard (2015a) with regard to the evolutions of the overall shear stress, the porosity, the orientation of the void and the magnitude of its principal axes were more or less well reproduced, the overall agreement being quite acceptable. The model was found in particular to satisfactorily account for (i) the large influence, prior to mesoscopic strain localization, of the aspect ratio of elliptic cylindrical voids upon the softening they generate; (ii) the occurrence of mesoscopic strain localization accelerating softening, even in the absence of significant void growth.

The ML model thus appears as a viable, more complete and versatile although more complex, alternative to Nahshon and Hutchinson (2008)'s proposed modification of Gurson (1977)'s model for the description of ductile rupture at low triaxialities.

This work opens perspectives of two kinds:

- The simulations depicted in the present paper represent only elementary tests of the ML model based on comparison with independent micromechanical simulations of the behavior of small homogeneous volumes. The model, having successfully passed the tests, must now be applied to full experimental specimens or industrial structures involving inhomogeneous stress states. This is all the more indispensable since the "reference" micromechanical simulations, in spite of their interest, do not cover the whole range of possible void shapes and loadings to be encountered in practice. A first example of application, pertaining to the simulation of fracture tests performed by Dunand and Mohr (2011a,b) on "butterfly specimens" subjected to combined tension and shear, will be presented shortly. Other future examples could include simulations of fracture tests of axisymmetric specimens subjected to combined tension and torsion.

- Also, the ML model may now be used to assist the development of improved, micromechanically-based models of (void coalescence)/(mesoscopic strain localization), along the lines recently defined by Benzerga and Leblond (2014). Indeed in models of this kind, the spacings of the voids in the different directions of space always have an important impact upon the onset of the phenomenon, generating a need for accurate prediction of the evolution of the void axes prior to this event. The ML model may be used for this purpose, quite in the same way as Pardoen and Hutchinson (2000) used the simpler GLD model in cases where the voids could be assumed to remain spheroidal. Conversely, the ML model may in turn benefit from the development of such micromechanical descriptions of (void coalescence)/(mesoscopic strain localization), which will serve as advantageous alternatives to the simple heuristic modelling proposed in this paper, based on a "critical value" of the parameter $f+g$ characterizing ductile damage.

\section{References}

Bao Y., Wierzbicki T. (2004). On fracture locus in the equivalent strain and stress triaxiality space. Int. J. Mech. Sci., 46, 81-98.

Barsoum I., Faleskog J. (2007). Rupture mechanisms in combined tension and shear Experiments. Int. J. Solids Struct., 44, 1768-1786. 
Barsoum I., Faleskog J. (2011). Micromechanical analysis on the influence of the Lode parameter on void growth and coalescence. Int. J. Solids Struct., 48, 925-938.

Beese A.M., Luo M., Li Y., Bai Y., Wierzbicki T. (2010). Partially coupled anisotropic fracture model for aluminum sheets. Engng. Fract. Mech., 77, 1128-1152.

Benzerga A., Leblond J.B. (2010). Ductile fracture by void growth to coalescence. Adv. Appl. Mech., 44, 169-305.

Benzerga A., Leblond J.B. (2014). Effective yield criterion accounting for microvoid coalescence. ASME J. Appl. Mech., 81, 031009-1 to 7.

Dahl J., Nielsen K.L., Tvergaard V. (2012). Effect of contact conditions on void coalescence at low stress triaxiality shearing. ASME J. Appl. Mech., 79, 021003-1 to 7.

de Borst R., Feenstra P.H. (1990). Studies in anisotropic plasticity with reference to the Hill criterion. Int. J. Numer. Meth. Engng., 29, 315-336.

Dunand M., Mohr D. (2011a). Optimized butterfly specimen for the fracture testing of sheet materials under combined normal and shear loading. Engng. Fract. Mech., 78, 2919-2934.

Dunand M., Mohr D. (2011b). On the predictive capabilities of the shear modified Gurson and the modified Mohr-Coulomb fracture models over a wide range of stress triaxialities and Lode angles. J. Mech. Phys. Solids, 59, 1374-1394.

Enakoutsa K., Leblond J.B., Perrin G. (2007). Numerical implementation and assessment of a phenomenological nonlocal model of ductile rupture. Comput. Meth. Appl. Mech. Engng., 196, 1946-1957.

Eshelby J. (1957). The determination of the elastic field of an ellipsoidal inclusion, and related problems. Proc. Roy. Soc. London A, 241, 376-396.

Garajeu M., Michel J.C., Suquet P. (2000). A micromechanical approach of damage in viscoplastic materials by evolution in size, shape and distribution of voids. Comput. Meth. Appl. Mech. Engng., 183, 223-246.

Ghahremaninezhad A., Ravi-Chandar K. (2013). Ductile failure in polycrystalline aluminum alloy $\mathrm{Al}$ 6061-T6 under shear dominant loading. Int. J. Fract., 180, 23-39.

Gologanu M. (1997). Etude de quelques problèmes de rupture ductile des métaux. Ph.D. Thesis, Université Pierre et Marie Curie (Paris 6) (in French).

Gologanu M., Leblond J.B., Devaux J. (1993). Approximate models for ductile metals containing non-spherical voids - Case of axisymmetric prolate ellipsoidal cavities. $J$. Mech. Phys. Solids, 41, 1723-1754.

Gologanu M., Leblond J.B., Devaux J. (1994). Approximate models for ductile metals containing non-spherical voids - Case of axisymmetric oblate ellipsoidal cavities. ASME J. Engng. Mater. Technol., 116, 290-297.

Gologanu M., Leblond J.B., Perrin G., Devaux J. (1997). Recent extensions of Gurson's model for porous ductile metals. In: Continuum Micromechanics, P. Suquet, ed., Springer-Verlag, New-York, pp. 61-130.

Gurson A.L. (1977). Continuum theory of ductile rupture by void nucleation and growth: Part I - Yield criteria and flow rules for porous ductile media. ASME J. Engng. Mater. Technol., 99, 2-15.

Halphen B. and Nguyen Q.S. (1975). Sur les matriaux standards généralisés. J. Mécanique, 14, 39-63 (in French).

Haltom S.S., Kyriakides S., Ravi-Chandar K. (2013). Ductile failure under combined shear and tension. Int. J. Solids Struct., 50, 1507-1522.

Hill R. (1948). A theory of the yielding and plastic flow of anisotropic metals. Proc. Roy. 
Soc. London A, 193, 281-297.

Huang Y. (1991). Accurate dilatation rates for spherical voids in triaxial stress fields. ASME J. Appl. Mech., 58, 1084-1086.

Kailasam M., Ponte-Castaneda P. (1998). A general constitutive theory for linear and nonlinear particulate media with microstructure evolution. J. Mech. Phys. Solids, 46, 427-465.

Koplik J., Needleman A. (1988). Void growth and coalescence in porous plastic solids. Int. J. Solids Struct., 24, 835-853.

Leblond J.B. (2015). UMAT available at the address www.dalembert.upmc.fr/home/ leblond/.

Leblond J.B., Gologanu M. (2008). External estimate of the yield surface of an arbitrary ellipsoid containing a confocal void. Comptes-Rendus Mécanique, 336, 813-819.

Leblond J.B., Mottet G. (2008). A theoretical approach of strain localization within thin planar bands in porous ductile materials. Comptes-Rendus Mécanique, 336, 176-189.

Madou K., Leblond J.B. (2012a). A Gurson-type criterion for porous ductile solids containing arbitrary ellipsoidal voids - I: Limit-analysis of some representative cell. J. Mech. Phys. Solids, 60, 1020-1036.

Madou K., Leblond J.B. (2012b). A Gurson-type criterion for porous ductile solids containing arbitrary ellipsoidal voids - II: Determination of yield criterion parameters. $J$. Mech. Phys. Solids, 60, 1037-1058.

Madou K., Leblond J.B. (2013). Numerical studies of porous ductile materials containing arbitrary ellipsoidal voids - I: Yield surfaces of representative cells. Eur. J. Mech. A/Solids, 42, 480-489.

Madou K., Leblond J.B., Morin L. (2013). Numerical studies of porous ductile materials containing arbitrary ellipsoidal voids - II: Evolution of the length and orientation of the void axes. Eur. J. Mech. A/Solids, 42, 490-507.

Morin L., Madou K., Leblond J.B., Kondo D. (2014). A new technique for finite element limit-analysis of Hill materials, with an application to the assessment of criteria for anisotropic plastic porous solids. Int. J. Engng. Sci., 74, 65-79.

Nahshon K., Hutchinson J.W. (2008). Modification of the Gurson model for shear failure. Eur. J. Mech./A Solids, 27, 1-17.

Nguyen Q.S. (1977). On the elastic plastic initial-boundary value problem and its numerical integration. Int. J. Num. Meth. Engng., 11, 817-832.

Nielsen K.L., Dahl J., Tvergaard V. (2012). Collapse and coalescence of spherical voids subject to intense shearing: studied in full 3D. Int. J. Fract., 177, 97-108.

Pardoen T., Hutchinson J.W. (2000). An extended model for void growth and coalescence. J. Mech. Phys. Solids, 48, 2467-2512.

Perrin G. (1992). Contribution à l'étude théorique et numérique de la rupture ductile des métaux. Thèse de Doctorat d'Etat, Ecole Polytechnique (in French).

Perrin G., Leblond J.B. (1990). Analytical study of a hollow sphere made of porous plastic material and subjected to hydrostatic tension - Application to some problems in ductile fracture of metals. Int. J. Plast., 6, 677-699.

Ponte-Castaneda P., Zaidman M. (1994). Constitutive models for porous materials with evolving microstructure. J. Mech. Phys. Solids, 42, 1459-1492.

Rice J.R., Tracey D.M. (1969). On the ductile enlargement of voids in triaxial stress fields. J. Mech. Phys. Solids, 17, 201-217.

Scheyvaerts F., Onck P., Tekoglu C., Pardoen T. (2011). The growth and coalescence 
of ellipsoidal voids in plane strain under combined shear and tension. J. Mech. Phys. Solids, 59, 373-397.

Siruguet K., Leblond J.B. (2004a). Effect of void locking by inclusions upon the plastic behavior of porous ductile solids - I: Theoretical modelling and numerical study of void growth. Int. J. Plast., 20, 225-254.

Siruguet K., Leblond J.B. (2004b). Effect of void locking by inclusions upon the plastic behavior of porous ductile solids - II: Theoretical modelling and numerical study of void coalescence. Int. J. Plast., 20, 255-268.

Song D., Agoras M., Ponte-Castaneda P. (2015). The evolution of pore shape and orientation in plastically deforming metals: implications for macroscopic response and shear localization. Mech. Mater., 90, 47-68.

Tvergaard V. (1981). Influence of voids on shear band instabilities under plane strain conditions. Int. J. Fract., 17, 389-407.

Tvergaard V. (1990). Material failure by void growth to coalescence. Adv. Appl. Mech., 27, 83-151.

Tvergaard V. (2008). Shear deformation of voids with contact modeled by internal pressure. Int. J. Mech. Sci., 50, 1459-1465.

Tvergaard V. (2009). Behaviour of voids in a shear field. Int. J. Fract., 158, 41-49.

Tvergaard V. (2012). Effect of stress-state and spacing on voids in a shear-field. Int. J. Solids Struct., 49, 3047-3054.

Tvergaard V. (2015a). Effect of initial void shape on ductile failure in a shear field. Mech. Mater., 90, 2-9.

Tvergaard V. (2015b). Behaviour of porous ductile solids at low stress triaxiality in different modes of deformation. Int. J. Solids Struct., 60-61, 28-34.

Tvergaard V., Needleman A. (1984). Analysis of the cup-cone fracture in a round tensile bar. Acta Metall., 32, 157-169.

Tvergaard V., Nielsen K.L. (2010). Relations between a micro-mechanical model and a damage model for ductile failure in shear. J. Mech. Phys. Solids, 58, 1243-1252.

Xue Z., Pontin M.A., Zok F.W., Hutchinson J.W. (2010). Calibration procedures for a computational model of ductile fracture. Engng. Fract. Mech., 77, 492-509. 